\title{
Symmetry-enforced topological band crossings in orthorhombic crystals: Classification and materials discovery
}

\author{
Andreas Leonhardt $\odot,{ }^{*}$ Moritz M. Hirschmann $\odot$, Niclas Heinsdorf, Xianxin Wu, \\ Douglas H. Fabini $\odot$, and Andreas P. Schnyder $\odot^{\dagger}$ \\ Max-Planck-Institut für Festkörperforschung, Heisenbergstrasse 1, D-70569 Stuttgart, Germany
}

(Received 13 August 2021; accepted 18 October 2021; published 6 December 2021)

\begin{abstract}
We identify all symmetry-enforced band crossings in nonmagnetic orthorhombic crystals with and without spin-orbit coupling and discuss their topological properties. We find that orthorhombic crystals can host a large number of different band degeneracies, including movable Weyl and Dirac points with hourglass dispersions, fourfold double Weyl points, Weyl and Dirac nodal lines, almost movable nodal lines, nodal chains, and topological nodal planes. Interestingly, spin-orbit coupled materials in space groups $18,36,44,45$, and 46 can have band pairs with only two Weyl points in the entire Brillouin zone. This results in simpler connectivity of the Fermi arcs and more pronounced topological responses than in materials with four or more Weyl points. In addition, we show that the symmetries of space groups 56,61 , and 62 enforce nontrivial weak $\mathbb{Z}_{2}$ topology in materials with strong spin-orbit coupling, leading to helical surface states. With these classification results in hand, we perform extensive database searches for orthorhombic materials crystallizing in the relevant space groups. We find that $\mathrm{Sr}_{2} \mathrm{Bi}_{3}$ and $\mathrm{Ir}_{2} \mathrm{Si}$ have bands crossing the Fermi energy with a symmetry-enforced nontrivial $\mathbb{Z}_{2}$ invariant, CuIrB possesses nodal chains near the Fermi energy, $\mathrm{Pd}_{7} \mathrm{Se}_{4}$ and $\mathrm{Ag}_{2} \mathrm{Se}$ exhibit fourfold double Weyl points, the latter one even in the absence of spin-orbit coupling, whereas the fourfold degeneracies in AuTISb are made up from intersecting nodal lines. For each of these examples, we compute the ab initio band structures, discuss their topologies, and for some cases also calculate the surface states.
\end{abstract}

DOI: 10.1103/PhysRevMaterials.5.124202

\section{INTRODUCTION}

The discovery of topological insulators more than ten years ago [1-3] has kicked off a classification program of topological band structures [4-6], which is still ongoing today. Insulators as well as Dirac and Weyl-type semimetals have been classified, both in terms of nonspatial symmetries [4,5] and crystalline symmetries [7-10]. The methods used to establish these classifications are wide ranging, including Clifford algebra extensions [5,11-14], minimal Dirac models [8,9], $\mathrm{K}$ theory [5], compatibility relations between irreducible representations [15-18], symmetry eigenvalue analyses [17-25], symmetry-based indicators [26], as well as the study of elementary band representations [27,28]. These classification works lay down the basis for the study of topological systems in general and can be used as guiding principles for the design and discovery of new topological systems. Indeed, when combined with materials databases, such as the ICSD from FIZ Karlsruhe [29] and the Materials Project database [30,31], these classifications have lead to the prediction of new topological materials [17-19,26,32,33].

\footnotetext{
*a.leonhardt@fkf.mpg.de

†a.schnyder@fkf.mpg.de
}

Published by the American Physical Society under the terms of the Creative Commons Attribution 4.0 International license. Further distribution of this work must maintain attribution to the author(s) and the published article's title, journal citation, and DOI. Open access publication funded by the Max Planck Society.
Topological materials are not only of fundamental interest, but are also sought after for their functional properties that can be harnessed for applications [34]. For example, the spin-momentum locking of the surface states could be used for low-dissipation transport in future post-silicon devices $[35,36]$. Moreover, the high mobility and large magnetoresistance of many Weyl semimetals could be useful for future high-speed electronics and spintronics [10,37]. Hence, there is a need for new topological materials with these properties. One strategy to discover new topological semimetals is to focus on symmetry-enforced topologies, i.e., topological features that are enforced to exist by symmetry alone, independent of the band dispersion, orbital content, and chemical composition of the material. Once the space group (SG) symmetries that enforce the desired band topologies are identified, a suitable material can be found by browsing databases of known materials by SG number.

Previously, we have applied this strategy to hexagonal, trigonal, and tetragonal crystals [17-19]. These investigations have uncovered, among other things, the accordion states in trigonal Te [38], and identified ZrIrSn and $\mathrm{NaSn}_{5}$ as nodal line materials with twofold and fourfold Weyl lines, respectively $[17,19]$. In this paper, we continue this classification program by investigating symmetry-enforced band crossings in orthorhombic crystals. Our results are summarized in Tables I-IV, which classify all symmetry-enforced topological features in band structures of orthorhombic crystals with time-reversal symmetry.

Tables I and III apply to band structures with time-reversal symmetry that squares to +1 , e.g., to electronic bands without 
TABLE I. Symmetry-enforced band crossings in spinless band structures of noncentrosymmetric orthorhombic SGs. The label of highsymmetry points and axes corresponds to the BZs shown in Fig. 1. The second column shows all pointlike degeneracies. They can be on a high-symmetry point (e.g., $\Gamma$ ), somewhere on an axis connecting two high-symmetry points (e.g., $\Gamma$-Z) or on one of several axes (e.g., $\Gamma-[X \underline{\vee} \underline{\vee Z}])$ with $\underline{\vee}$ indicating the exclusive OR. Numbers in brackets indicate the number of bands involved. The topological type of the degeneracies are listed under notable features if it is different from a Weyl point. The third column lists all symmetry-enforced nodal lines. High-symmetry lines are specified by the two TRIMs they connect according to Fig. 1, intersecting nodal lines are contracted, e.g., T-Z-U specifies two nodal lines on $\mathrm{Z}-\mathrm{U}=(u, 0, \pi)$ and $\mathrm{Z}-\mathrm{T}=(0, v, \pi)$. The notation $(A ; B)$ indicates an hourglass nodal line between two pointor linelike degeneracies $A$ with eigenvalue pairing $(+,-)$ and $B$ with $(+,+)$ or $(-,-)$. The fourth column lists all twofold degenerate nodal planes defined through Cartesian coordinates as shown in Fig. 1. The splitting of bands is linear to lowest order in momentum for all types of degeneracies.

\begin{tabular}{|c|c|c|c|c|}
\hline SG & Points & Lines & Nodal planes & Notable features \\
\hline \multicolumn{5}{|l|}{$16(P 222)$} \\
\hline $17\left(P 222_{1}\right)$ & & & $k_{z}=\pi$ & \\
\hline $18\left(P 2{ }_{1} 2{ }_{1}\right)$ & & & $k_{x / y}=\pi$ & \\
\hline $19\left(P 2{ }_{1} 2_{1} 2_{1}\right)$ & $\Gamma-[X \bigvee Y \vee Z](4), R(4)$ & & $k_{x / y / z}=\pi$ & fourfold double Weyl point \\
\hline $20\left(C 222_{1}\right)$ & & & $k_{z}=\pi$ & \\
\hline \multicolumn{5}{|l|}{$21(C 222)$} \\
\hline \multicolumn{5}{|l|}{$22(F 222)$} \\
\hline \multicolumn{5}{|l|}{$23(I 222)$} \\
\hline $24\left(I 2_{1} 2_{1} 2_{1}\right)$ & $\Gamma-[X \vee Y \vee Z Z](2), W$ & & & only four Weyl points \\
\hline \multicolumn{5}{|l|}{$25($ Pmm 2$)$} \\
\hline $26\left(P m c 2_{1}\right)$ & & & $k_{z}=\pi$ & \\
\hline $27(P c c 2)$ & & Z-U-R-T-Z & & \\
\hline $28($ Pma2) & & X-U-R-S-X & & \\
\hline $29\left(\mathrm{Pca}_{1}\right)$ & & $(U-\mathrm{X}-\mathrm{S}-R ; U-R)$ & $k_{z}=\pi$ & \\
\hline $30($ Pnc2) & & Z-U-R-S-Y-T-Z & & \\
\hline $31\left(P m n 2_{1}\right)$ & & U-X-S-R & $k_{z}=\pi$ & \\
\hline $32(P b a 2)$ & & X-U-R-T-Y-S-X & & \\
\hline $33\left(\right.$ Pna $\left._{1}\right)$ & & $(\mathrm{S}-\mathrm{X}-U ; U-\mathrm{R}), \mathrm{S}-\mathrm{Y}-\mathrm{T}$ & $k_{z}=\pi$ & \\
\hline $34($ Pnn2) & & X-S-Y-T-Z-U-X & & \\
\hline \multicolumn{5}{|l|}{$35(\mathrm{Cmm} 2)$} \\
\hline $36\left(C m c 2_{1}\right)$ & & & $k_{z}=\pi$ & \\
\hline $37(C c c 2)$ & & U-Z-T & & \\
\hline \multicolumn{5}{|l|}{$38(A m m 2)$} \\
\hline $39($ Aem 2$)$ & & $\mathrm{R}-\mathrm{S}$ & & \\
\hline 40 (Ama2) & & A-Z-T & & \\
\hline 41 (Aea2) & & R-S, A-Z-T & & \\
\hline \multicolumn{5}{|l|}{$42(F m m 2)$} \\
\hline $43(F d d 2)$ & & A-Z-T-Y & & \\
\hline \multicolumn{5}{|l|}{$44(\operatorname{Imm} 2)$} \\
\hline $45(I b a 2)$ & & R-W-S & & \\
\hline $46(\operatorname{Ima2})$ & & R-W & & \\
\hline
\end{tabular}

spin-orbit coupling (SOC). This type of time-reversal symmetry is present in materials with light elements, where SOC can be neglected. It also occurs in the excitation spectra of bosonic quasiparticles, e.g., phonon or magnon bands $[39,40]$, and in synthetic materials, such as photonic crystals [41] and electric circuit networks $[42,43]$. We find that without SOC the band structures can exhibit movable Weyl points, due to screw rotations, movable and almost movable nodal lines, due to mirror symmetries, and nodal planes, due to the combination of screw rotations with time-reversal symmetry. By the bulk-boundary correspondence, the Weyl points and nodal lines lead to Fermi arc and drumhead surface states, respectively.

Tables II and IV list all symmetry-enforced topological features in band structures with time-reversal symmetry that squares to -1 , e.g., in electronic bands with strong SOC. We find that with SOC the bands can possess various types of different point degeneracies, namely, Kramers-Weyl points, fourfold points with zero Chern number $(\mathcal{C}=0)$, movable Weyl and Dirac points with hourglass dispersions, and fourfold double Weyl points with $|\mathcal{C}|=2$. Line degeneracies in the presence of SOC also exist in different varieties: (almost) movable Weyl lines with hourglass dispersion, Dirac lines protected by off-centered symmetries, and nodal chains. Finally, there are nine SGs with nodal planes at the BZ boundary, of which the chiral SGs $17-19$ and 20 can have a nonzero topological charge. Remarkably, in SG 19 the nodal planes must always be topological by symmetry, irrespective of the orbital content and dispersions of the bands (Sec. IV C).

Furthermore, we identify two SGs, namely, 56 and 62, which in the presence of SOC exhibit symmetry-enforced nontrivial weak $\mathbb{Z}_{2}$ invariants (Sec. VIG). The same holds for SG 61, assuming that elementary band representations with different inversion eigenvalues do not mix. Similarly, SGs 52 
TABLE II. Symmetry-enforced band crossings in band structures with SOC of noncentrosymmetric orthorhombic SGs. The notation is identical to the one in Table I. Additionally, almost movable lines are indicated by $(A ;-)$ with $A$ being the high-symmetry point they run through as described in Sec. VE. The splitting of bands is linear to lowest order in momentum for all types of degeneracies.

\begin{tabular}{|c|c|c|c|c|}
\hline SG & Points & Lines & Nodal planes & Notable features \\
\hline $16(P 222)$ & all TRIMs & & & \\
\hline $17\left(P 222_{1}\right)$ & $\begin{array}{c}\Gamma, \mathrm{X}, \mathrm{Y}, \mathrm{S}, \\
\Gamma-\mathrm{Z}(4), \mathrm{X}-\mathrm{U}(4), \\
\mathrm{Y}-\mathrm{T}(4), \mathrm{S}-\mathrm{R}(4)\end{array}$ & & $k_{z}=\pi$ & only four Weyl points \\
\hline $18\left(P 2_{1} 2_{1} 2\right)$ & $\begin{array}{c}\Gamma, \mathrm{Z}, \Gamma-\mathrm{X}(4), \\
\Gamma-\mathrm{Y}(4), \mathrm{Z}-\mathrm{U}(4), \\
\mathrm{Z}-\mathrm{T}(4), \mathrm{S}(4), \\
\mathrm{R}(4)\end{array}$ & & $k_{x / y}=\pi$ & $\begin{array}{l}\text { fourfold double Weyl points ( } n \text { even), only } \\
\text { two Weyl points ( } n \text { odd) }\end{array}$ \\
\hline $19\left(P 2_{1} 2_{1} 2_{1}\right)$ & $\begin{array}{c}\Gamma, \Gamma-\mathrm{Z}(4), \\
\Gamma-\mathrm{X}(4), \Gamma-\mathrm{Y}(4), \\
\mathrm{S}(4), \mathrm{T}(4), \mathrm{U}(4), \\
\mathrm{R}-[\mathrm{S} \underline{\mathrm{V}} \mathrm{T} \underline{\mathrm{V}}](8)\end{array}$ & & $k_{x / y / z}=\pi$ & $\begin{array}{l}\text { top. nodal plane trio, fourfold double Weyl } \\
\text { points }\end{array}$ \\
\hline $20\left(C 222_{1}\right)$ & $\begin{array}{c}\Gamma, \mathrm{S}, \mathrm{Y}, \Gamma-\mathrm{Z}(4) \\
\mathrm{Y}-\mathrm{T}(4), \mathrm{S}-\mathrm{R}(4)\end{array}$ & & $k_{z}=\pi$ & only four Weyl points \\
\hline $21(C 222)$ & all TRIMs & & & \\
\hline $22(F 222)$ & all TRIMs & & & \\
\hline $23(I 222)$ & all TRIMs, W & & & \\
\hline $24\left(I 2_{1} 2_{1} 2_{1}\right)$ & $\begin{array}{c}\text { all TRIMs, } \\
\mathrm{W}-[\mathrm{R} \vee \mathrm{S} \underline{\vee}] \mathrm{T}(4)\end{array}$ & & & \\
\hline $25($ Pmm2) & & $\Gamma-\mathrm{Z}, \mathrm{X}-\mathrm{U}, \mathrm{Y}-\mathrm{T}, \mathrm{S}-\mathrm{R}$ & & \\
\hline $26\left(P m c 2_{1}\right)$ & & $(\Gamma-Z, \mathrm{Y}-T ; Z-T),(\mathrm{X}-U, R-\mathrm{S} ; U-R)$ & $k_{z}=\pi$ & \\
\hline $27(P c c 2)$ & $\begin{array}{l}\mathrm{Z}(4), \mathrm{T}(4) \\
\mathrm{U}(4), \mathrm{R}(4)\end{array}$ & Г-Z-U-R-T-Z, X-U, S-R, Y-T & & fourfold points $(\mathcal{C}=0)$ \\
\hline $28(P m a 2)$ & & $(\Gamma-\mathrm{Z} ; \mathrm{X}, \mathrm{U}),(\mathrm{Y}-\mathrm{T} ; \mathrm{S}, \mathrm{R}), \mathrm{X}-\mathrm{S}, \mathrm{U}-\mathrm{R}$ & & \\
\hline $29\left(\mathrm{Pca2}_{1}\right)$ & & $\begin{array}{c}(\Gamma-Z ; Z-\mathrm{U}, \mathrm{X}),(\mathrm{Y}-T ; \mathrm{S}, \mathrm{R}-T) \\
(\mathrm{X}-\mathrm{S} ; \mathrm{U}-\mathrm{R})\end{array}$ & $k_{z}=\pi$ & \\
\hline $30(P n c 2)$ & $\mathrm{Z}(4), \mathrm{U}(4)$ & $\begin{array}{c}(\Gamma-Z-\mathrm{T} ; \mathrm{Y}),(\mathrm{X}-U-\mathrm{R} ; \mathrm{S}),(\mathrm{Y}-\mathrm{S} ; \mathrm{T}, \mathrm{R}) \\
\mathrm{Z}-\mathrm{U}\end{array}$ & & nodal chain, fourfold points $(\mathcal{C}=0)$ \\
\hline $31\left(P m n 2_{1}\right)$ & & $\begin{array}{c}(\Gamma-Z, T-\mathrm{Y} ; Z-T),(\Gamma-Z-\mathrm{U} ; \mathrm{X}) \\
(\mathrm{Y}-T-\mathrm{R} ; \mathrm{S}), \mathrm{X}-\mathrm{S}\end{array}$ & $k_{z}=\pi$ & \\
\hline $32($ Pba2) & $\mathrm{R}(4), \mathrm{S}(4)$ & $\begin{array}{c}(\Gamma-\mathrm{Z} ; \mathrm{Y}, \mathrm{T}),(\Gamma-\mathrm{Z} ; \mathrm{X}, \mathrm{T}), \mathrm{X}-\mathrm{S}-\mathrm{Y} \\
\mathrm{U}-\mathrm{R}-\mathrm{T}, \mathrm{S}-\mathrm{R}\end{array}$ & & fourfold points $(\mathcal{C}=0)$ \\
\hline $33\left(\right.$ Pna $\left._{1}\right)$ & $S(4)$ & $\begin{array}{c}(R-S-\mathrm{X} ; R-\mathrm{U}),(\Gamma-Z ; Z-\mathrm{U}, \mathrm{X}) \\
(\Gamma-Z-\mathrm{T} ; \mathrm{Y}), \mathrm{Y}-\mathrm{S}\end{array}$ & $k_{z}=\pi$ & fourfold point $(\mathcal{C}=0)$ \\
\hline $34($ Pnn2) & $\mathrm{Z}(4), \mathrm{S}(4)$ & $\begin{array}{c}(\Gamma-Z-\mathrm{T} ; \mathrm{Y}),(\mathrm{Y}-S-\mathrm{R} ; \mathrm{T}),(\Gamma-Z-\mathrm{U} ; \mathrm{X}) \\
(\mathrm{X}-\mathrm{S}-\mathrm{R} ; \mathrm{U})\end{array}$ & & nodal chain, fourfold points $(\mathcal{C}=0)$ \\
\hline $35(\mathrm{Cmm} 2)$ & $\mathrm{R}, \mathrm{S}$ & $\Gamma-\mathrm{Z}, \mathrm{Y}-\mathrm{T}$ & & only four Weyl points \\
\hline $36\left(C m c 2_{1}\right)$ & S, R-S(4), & $(\Gamma-Z, Y-T ; Z-T)$ & $k_{z}=\pi$ & only two (four) Weyl points for $n$ odd (even) \\
\hline $37(C c c 2)$ & $\mathrm{R}, \mathrm{S}, \mathrm{Z}(4), \mathrm{T}(4)$ & $\Gamma-\mathrm{Z}-\mathrm{T}-\mathrm{Y}, \mathrm{Z}-\mathrm{U}$ & & fourfold points $(\mathcal{C}=0)$, only four Weyl points \\
\hline $38(A m m 2)$ & & $(\mathrm{R} ;-),(\mathrm{S} ;-), \Gamma-\mathrm{X}, \mathrm{Z}-\mathrm{A}$ & & \\
\hline 39 (Aem2) & & $(\Gamma-\mathrm{X} ; \mathrm{S}),(\mathrm{Z}-\mathrm{A} ; \mathrm{R}), \mathrm{R}-\mathrm{S}$ & & \\
\hline $40($ Ama2) & & $(\Gamma-\mathrm{X} ; \mathrm{Z}, \mathrm{T}),(\mathrm{R} ;-),(\mathrm{S} ;-), \mathrm{Z}-\mathrm{T}$ & & \\
\hline $41($ Aea2) & & $(\Gamma-X ; Z, T),(\Gamma-X ; S),(Z-T ; R), R-S$ & & \\
\hline $42(F m m 2)$ & $\mathrm{L}$ & $\Gamma-\mathrm{Z}, \mathrm{Y}-\mathrm{T}$ & & only four Weyl points \\
\hline $43(F d d 2)$ & $\mathrm{L}, \mathrm{Z}(4)$ & $(\Gamma-Z-\mathrm{T} ; \mathrm{Y})_{k_{x}=0},(\Gamma-\mathrm{A}-\mathrm{Y} ; \mathrm{T})_{k_{y}=0}$ & & $\begin{array}{l}\text { nodal chain, fourfold point, only four Weyl } \\
\text { points }\end{array}$ \\
\hline $44(\operatorname{Imm} 2)$ & $\mathrm{T}$ & $(\mathrm{R} ;-),(\mathrm{S} ;-), \Gamma-\mathrm{Z}$ & & only two Weyl points \\
\hline 45 (Iba2) & $\mathrm{T}, \mathrm{T}-\mathrm{W}(4)$ & $(\Gamma-Z ; S, R), \mathrm{R}-\mathrm{W}-\mathrm{S}$ & & only two (four) Weyl points for $n$ odd (even) \\
\hline $46(\operatorname{Ima2})$ & $\mathrm{T}$ & $(\Gamma-\mathrm{Z} ; \mathrm{R}),(\mathrm{S} ;-), \mathrm{R}-\mathrm{W}$ & & only two Weyl points \\
\hline
\end{tabular}

and 60 have nontrivial weak $\mathbb{Z}_{2}$ invariants, albeit only with weak SOC that does not induce band inversions. By the bulkboundary correspondence, spin-orbit coupled materials in all these SGs exhibit helical surface states, that could potentially be used for low-dissipation (spin) transport.
Lastly, we determine those SGs where the number of Weyl points formed by a spin-orbit coupled band pair can be as low as four (or even two), i.e., where only four (or two) of the eight time-reversal invariant momenta (TRIMs) host Weyl points, while the other TRIMs are part of nodal lines 
TABLE III. Symmetry-enforced band crossings in spinless band structures of centrosymmetric orthorhombic SGs. The notation is identical to the one introduced in Tables I and II. Fourfold degenerate nodal lines are indicated by (4). The splitting of bands is linear to lowest order in momentum for all types of degeneracies.

\begin{tabular}{|c|c|c|c|c|}
\hline SG & Points & Lines & Nodal planes & Notable features \\
\hline \multicolumn{5}{|l|}{$47($ Pmmm $)$} \\
\hline 48 (Pnnn) & & Z-U-X-S-Y-T-Z & & \\
\hline 49 (Pccm) & & Z-U-R-T-Z & & \\
\hline $50($ Pban $)$ & & X-U-R-T-Y-S-X & & \\
\hline $51($ Pmma $)$ & & & $k_{x}=\pi$ & \\
\hline $52($ Pnna) & & U-Z-T, X-S, (U-X-S;S-R), (X-S,Y-S) & $k_{y}=\pi$ & precedes unstable $\mathbb{Z}_{2}$ invariant (with SOC) \\
\hline 53 (Pmna) & & U-X-S-R & $k_{z}=\pi$ & \\
\hline $54(P c c a)$ & & $(\mathrm{Z}-U ; U-\mathrm{X}),(\mathrm{T}-R ; R-\mathrm{S}),(U-R ; R-\mathrm{T}-\mathrm{Z}-U)$ & $k_{x}=\pi$ & \\
\hline 55 (Pbam) & & & $k_{x}, k_{y}=\pi$ & \\
\hline $56(P c c n)$ & & $(T-\mathrm{Z} ; \mathrm{Y}-T),(U-\mathrm{Z}-T ; U-\mathrm{R}-T),(\mathrm{Z}-U ; U-\mathrm{X})$ & $k_{x}, k_{y}=\pi$ & precedes $\mathbb{Z}_{2}$ invariant (with SOC) \\
\hline $57(\mathrm{Pbcm})$ & & $\mathrm{R}-\mathrm{T}(4),(\mathrm{Y}-T ; T-\mathrm{Z}),(\mathrm{S}-R ; R-\mathrm{U})$ & $k_{y}, k_{z}=\pi$ & \\
\hline $58($ Pnnm $)$ & & $\mathrm{T}-\mathrm{Z}-\mathrm{U}$ & $k_{x}, k_{y}=\pi$ & \\
\hline $59($ Pmmn $)$ & & & $k_{x}, k_{y}=\pi$ & \\
\hline $60(P b c n)$ & & $\mathrm{U}-\mathrm{R}(4),(\mathrm{Y}-T ; T-\mathrm{Z}),(\mathrm{Z}-U ; U-\mathrm{X}),(R-\mathrm{S}-\mathrm{Y}-T ; T-R)$ & $k_{x}, k_{z}=\pi$ & precedes unstable $\mathbb{Z}_{2}$ invariant (with SOC) \\
\hline $61(P b c a)$ & & S-R(4), U-R-T(4), (Y-T;T-Z), (Z-U;U-X), (X-S;S-Y) & $k_{x / y / z}=\pi$ & \\
\hline $62($ Pnma $)$ & & $\mathrm{S}-\mathrm{R}(4),(\mathrm{X}-S ; S-\mathrm{Y})$ & $k_{x / y / z}=\pi$ & precedes $\mathbb{Z}_{2}$ invariant (with SOC) \\
\hline $63(\mathrm{Cmcm})$ & & & $k_{z}=\pi$ & \\
\hline $64(\mathrm{Cmca})$ & & $\mathrm{S}-\mathrm{R},(\mathrm{S} ;-)$ & $k_{z}=\pi$ & \\
\hline \multicolumn{5}{|l|}{$65(\mathrm{Cmmm})$} \\
\hline $66(\mathrm{Cccm})$ & & A-Z-T & & \\
\hline 67 (Cmme) & & $\mathrm{S}-\mathrm{R},(\mathrm{S} ;-),(\mathrm{R} ;-)$ & & \\
\hline $68($ Ccce $)$ & & A-Z-T, S-R, $(\mathrm{S} ;-),(\mathrm{R} ;-)$ & & \\
\hline \multicolumn{5}{|l|}{$69(F m m m)$} \\
\hline $70(F d d d)$ & & A-Z-T-Y & & \\
\hline \multicolumn{5}{|l|}{$71(\mathrm{Immm})$} \\
\hline $72($ Ibam $)$ & & S-W-R, $(\mathrm{S} ;-),(\mathrm{R} ;-)$ & & \\
\hline $73($ Ibca $)$ & $\mathrm{W}(4)$ & S-W-R, W-T, (S;-), (R;-), (T;-) & & fourfold point $(\mathcal{C}=0)$ at half filling \\
\hline $74($ Imma $)$ & & $\mathrm{W}-\mathrm{T},(\mathrm{T} ;-)$ & & \\
\hline
\end{tabular}

or nodal planes. Assuming that there are no other accidental Weyl points, band structures in these SGs have large Fermi arc surface states with a simple connectivity. Moreover, the topological responses, such as the anomalous (spin) Hall effect, are expected to be enhanced, since Weyl points with opposite chiralities are separated by a large distance in reciprocal space [44-46].

Using these classification results as an input, we perform an extensive database search for materials crystallizing in the relevant SGs (Sec. III and Fig. 2). This search yields six candidate materials, whose band topology we study using DFT calculations and by determining the irreducible representations at high-symmetry points. From this, we find that $\mathrm{Ag}_{2} \mathrm{Se}$ and $\mathrm{Pd}_{7} \mathrm{Se}_{4}$ possess fourfold double Weyl points and nodal planes (Sec. IV B 1). AuTlSb exhibits hourglass nodal lines and a fourfold point with $\mathcal{C}=0$, while CuIrB has nodal chains near the Fermi level. Both $\mathrm{Sr}_{2} \mathrm{Bi}_{3}$ and $\mathrm{Ir}_{2} \mathrm{Si}$ have bands crossing the Fermi level with nontrivial $\mathbb{Z}_{2}$ invariants.

The remainder of the paper is organized as follows. We start in Sec. II by defining our naming conventions and by describing the six different orthorhombic BZs and their TRIMs and high-symmetry lines. Our selection criteria for the database search are described in Sec. III, which yields six candidate materials. The band topologies and dispersions of these materials are presented in those sections where the corresponding SGs are discussed. In Sec. IV, we study the band topologies for the rhombic disphenoidal SGs (SGs 1624), which are chiral due to the absence of both mirror and inversion symmetries. Sections V and VI contain the analyses of the rhombic pyramidal SGs (SGs 25-46) and the rhombic dipyramidal SGs (SGs 47-74), respectively. Some concluding remarks are given in Sec. VII. Additional $a b$ initio band structures of the example materials are presented in Appendix A. In Appendix B, we present low-energy models of the fourfold degenerate points discussed in the main text. Appendix $\mathrm{C}$ contains a minimal tight-binding model for SG 19, which highlights the enforced features of chiral orthorhombic SGs both with and without SOC.

\section{CONVENTIONS}

\section{A. Symmetries}

The orthorhombic SGs consist of all those SGs with only twofold symmetries on the three mutual orthogonal symmetry axes. Those symmetries are twofold (screw) rotations and (glide) mirror symmetries. In case of three orthogonal mirror symmetries, there is also inversion symmetry. Additionally, we always assume time-reversal symmetry to be present. Since we will make frequent use of these symmetries, we introduce the notation with some examples by explicitly writing out the transformed coordinates of a point $(x, y, z)$ in Cartesian 
TABLE IV. Symmetry-enforced band crossings in band structures with SOC of centrosymmetric orthorhombic SGs. The notation is identical to the ones introduced in Tables I and II. All degeneracies are linearly dispersing Dirac points and lines and the overall twofold degeneracy due to $\mathcal{P} \mathcal{T}$ is not counted in the number of involved bands. In the fourth column, we list planes with a nontrivial $\mathbb{Z}_{2}$ invariant via the TRIMs they contain of which the TRIM with identical inversion eigenvalue pairing is shown in bold.

\begin{tabular}{|c|c|c|c|c|}
\hline SG & Points & Lines & Nontrivial planes & Notable features \\
\hline \multicolumn{5}{|l|}{$47($ Pmmm $)$} \\
\hline $48($ Pnnn $)$ & $\mathrm{S}, \mathrm{T}, \mathrm{U}, \mathrm{X}, \mathrm{Y}, \mathrm{Z}$ & & & \\
\hline $49($ Pccm $)$ & $\mathrm{R}, \mathrm{T}, \mathrm{U}, \mathrm{Z}$ & & & \\
\hline $50($ Pban $)$ & $\mathrm{R}, \mathrm{S}, \mathrm{T}, \mathrm{U}, \mathrm{X}, \mathrm{Y}$ & & & \\
\hline $51(P m m a)$ & & U-X, S-R & & \\
\hline $52($ Pnna $)$ & $\mathrm{U}, \mathrm{X}, \mathrm{Z}, \mathrm{X}-\mathrm{S}(4)$ & R-S-Y & ZURT, XUYT & movable Dirac point \\
\hline $53($ Pmna $)$ & $\mathrm{X}, \mathrm{S}$ & $\mathrm{T}-\mathrm{Z}$ & & \\
\hline $54($ Pcca $)$ & $\mathrm{T}, \mathrm{Z}, \mathrm{U}-\mathrm{Z}(4), \mathrm{R}-\mathrm{T}(4)$ & S-R-U-X & & \\
\hline $55($ Pbam $)$ & & X-S-R-U, S-Y, R-T & & \\
\hline $56(P c c n)$ & Z, Z-U(4), Z-T(4) & Y-T-R-U-X, S-R & XUSR, YTSR & movable Dirac points \\
\hline $57(\mathrm{Pbcm})$ & & U-R, T-Z, (S-Y;R,T) & & only Dirac nodal line at half filling \\
\hline $58($ Pnnm $)$ & $\mathrm{Z}$ & X-S-R, S-Y & & \\
\hline $59(P m m n)$ & & X-U, S-R, Y-T & & \\
\hline $60(P b c n)$ & $\mathrm{Y}, \mathrm{T}-\mathrm{Y}(4)$ & $(\mathrm{X}-\mathrm{U} ; \mathrm{R}),(\mathrm{R}-\mathrm{T}-\mathrm{Z} ; \mathrm{U})$ & XSTZ & Dirac nodal chain \\
\hline $61(P b c a)$ & & $(\mathrm{U}-\mathrm{X} ; \mathrm{S}),(\mathrm{S}-\mathrm{Y} ; \mathrm{T}),(\mathrm{T}-\mathrm{Z} ; \mathrm{U})$ & XYRZ & three Dirac nodal lines at half filling \\
\hline $62($ Pnma $)$ & & Z-U, R-T, S-Y, (R-U-X;S) & XUTY, ZURT & \\
\hline $63(\mathrm{Cmcm})$ & & $(\mathrm{R} ;-), \mathrm{Z}-\mathrm{T}$ & & \\
\hline $64(\mathrm{Cmca})$ & $\mathrm{S}$ & Z-A & & \\
\hline \multicolumn{5}{|l|}{$65(\mathrm{Cmmm})$} \\
\hline $66($ Cccm $)$ & $\mathrm{T}, \mathrm{Z}$ & & & \\
\hline 67 (Cmme) & $\mathrm{R}, \mathrm{S}$ & & & \\
\hline $68($ Ccce $)$ & $\mathrm{T}, \mathrm{Z}, \mathrm{R}, \mathrm{S}$ & & & \\
\hline \multicolumn{5}{|l|}{$69($ F mmm $)$} \\
\hline $70(F d d d)$ & $\mathrm{T}, \mathrm{Y}, \mathrm{Z}$ & & & \\
\hline \multicolumn{5}{|l|}{$71($ Immm $)$} \\
\hline $72($ Ibam $)$ & $\mathrm{R}, \mathrm{S}, \mathrm{W}$ & & & \\
\hline $73(I b c a)$ & $\mathrm{R}, \mathrm{S}, \mathrm{T}, \mathrm{W}-[\mathrm{R} \vee \mathrm{S} \underline{\vee} \mathrm{T}](4)$ & & & movable Dirac point \\
\hline $74($ Imma $)$ & $\mathrm{T}$ & & & \\
\hline
\end{tabular}

coordinates aligned with the conventional unit cell. Additionally, we include the action in spin space in terms of the Pauli matrices $\sigma_{i}, i \in\{x, y, z\}$, and $\sigma_{0}=\mathbb{1}_{2 \times 2}$. A translation by a lattice vector $(a, b, c)$ in real space is denoted by

$$
\begin{aligned}
& t(a, b, c): \\
& (x, y, z) \mapsto(x+a, y+b, z+c) \otimes \sigma_{0} .
\end{aligned}
$$

A twofold rotation about the $z$ axis with (fractional) translation vector $(a, b, c)$ is written as

$$
\begin{aligned}
& 2_{001}(a, b, c): \\
& (x, y, z) \mapsto(-x+a,-y+b, z+c) \otimes \mathrm{i} \sigma_{z} .
\end{aligned}
$$

Similarly, a mirror symmetry with $x z$ mirror planes and (fractional) translation vector $(a, b, c)$ is written as

$$
\begin{gathered}
M_{010}(a, b, c): \\
(x, y, z) \mapsto(x+a,-y+b, z+c) \otimes \mathrm{i} \sigma_{y} .
\end{gathered}
$$

For SGs with inversion symmetry $\mathcal{P}$, the origin choice is always at an inversion center (origin choice 2 in Ref. [47]),

$$
\mathcal{P}:(x, y, z) \mapsto(-x,-y,-z) \otimes \sigma_{0} .
$$

Finally, time-reversal symmetry is denoted by

$$
\mathcal{T}:(x, y, z) \mapsto(x, y, z) \otimes \mathrm{i} \sigma_{y} \mathcal{K} .
$$

Our analysis covers spinful and spinless band structures of the orthorhombic SGs. The spinful symmetry groups are the double $\mathrm{SG}$ with a distinguished element for a $2 \pi$ rotation, $\mathbb{1} \otimes\left(-\sigma_{0}\right)$.

Spinless representations are relevant for bosonic band structures, including in metamaterials, and materials with negligible spin-orbit coupling. In the latter case, we make use of the unbroken SU(2) symmetry. This allows us to use spinless representations by removing the spin part of the symmetries in Eqs.(2.1)-(2.5) using a properly chosen SU(2) rotation. This way each spin degree of freedom can be treated independently without the need of using double SGs. For our results in that case, we will not include the resulting spin degeneracy, unless it is mentioned explicitly. We use the parameter $\zeta=0,1$ to distinguish between the spinless and the spinful case, for example the representation of a $2 \pi$ rotation is always $(-1)^{\zeta}$.

\section{B. Orthorhombic lattices and Brillouin zones}

There are four Bravais lattices compatible with combinations of the above symmetries, primitive, side- or 


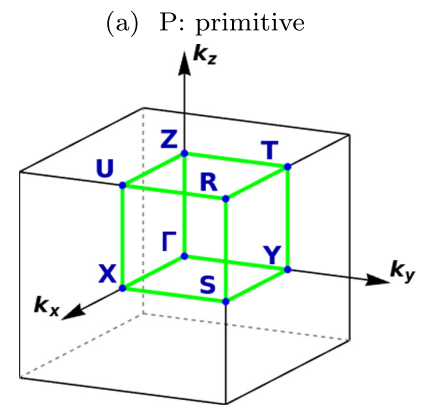

(b) C: base-centered

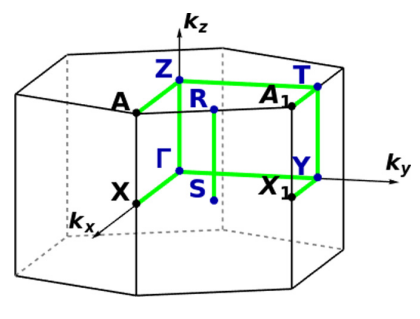

(c) F: face-centered

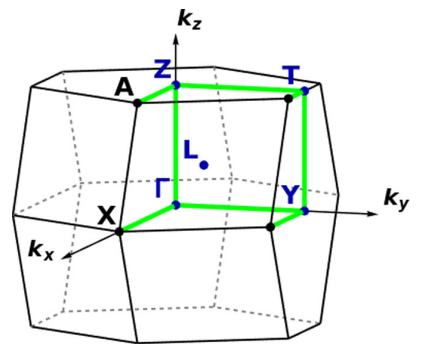

(d) I: body-centered

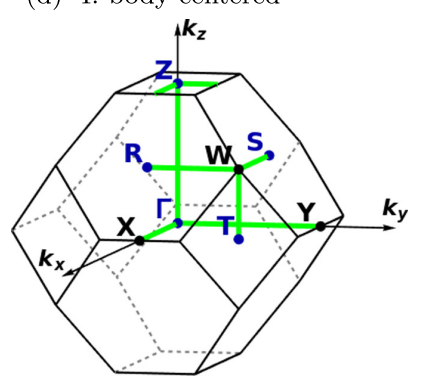

FIG. 1. Brillouin zones (BZs) for lattices with $a<b<c$ in the orthorhombic SGs. Of the several types in the face-centered case, the one for $\frac{1}{a^{2}}=\frac{1}{b^{2}}+\frac{1}{c^{2}}$ is shown. TRIMs are labeled in blue, rotation axes are shown in green and labeled by the points they are connecting.

base-centered, face-centered, and body-centered lattices. The conventional cells of all orthorhombic lattices have right angles and three independent lengths, $a, b$, and $c$. In the following, we will omit lengths by expressing points in terms of the reciprocal lattice vectors of the conventional lattice in the crystallographic definition, i.e., excluding the factor $2 \pi$.

Note that in the orthorhombic crystal system, there is no unique primary symmetry axis and there are various conventions in choosing the primary, secondary and tertiary symmetry directions. Since our analysis is based on symmetries alone, we align the symmetries according to their position in the Hermann-Mauguin symbols in the conventional setting [47] with the directions a, b and c. See the Hermann-Mauguin symbols in the first column of Tables I to IV for the assignment of symmetries to the crystal axes. This convention is also used by the Bilbao Crystallographic Server [16]. In high-throughput calculations, the axes are often ordered such that $a<b<c$ regardless of symmetry [48] and labels need to be interchanged accordingly to compare the results.

There are always eight TRIMs in the Brillouin zone (BZ). In the BZ of primitive lattices, these are also the points of maximal symmetry, i.e., their little group is identical to the SG and they are invariant under all symmetry operations. Figure 1(a) shows the BZ of primitive lattices, where all possible axes invariant under rotations are shown in green. An axis will be denoted by the two labeled points on it, e.g., $\Gamma-X$ for an axis parametrized by $(u, 0,0)$. Contractions are possible, i.e., the two axes $\Gamma-Z$ and Z-R are compactly written as $\Gamma-Z-R$.

In base- [side-] centered lattices, indicated by the lattice symbol $\mathrm{C}[\mathrm{A}]$, there is an additional lattice site at the center of the (001) [(100)] face. Only four TRIMs have maximal symmetry, $\Gamma, \mathrm{Z}, \mathrm{Y}$, and T. For the remaining TRIMs S and R there are two nonequivalent copies only invariant under symmetries of the third (first) symmetry axis and the remaining symmetries map the two copies onto each other, see Fig. 1(b). There are now two rotation axes connecting $\Gamma$ and $Y$, and we introduce the additional labels $\mathrm{X}$ and $\mathrm{A}$ to distinguish the axis $\Gamma-\mathrm{Y}=(0, v, 0)$ from $\Gamma-\mathrm{X}=(u, 0,0)$ and equivalently $\mathrm{Z}-\mathrm{T}=\left(0, v, \frac{\pi}{2}\right)$ and $\mathrm{Z}-\mathrm{A}=\left(u, 0, \frac{\pi}{2}\right)$. In the case $a>b$, the first BZ extends to $\mathrm{Y}$ in [100] direction and the additional points appear along [010]. Our results, however, hold always in relation to the orientation of symmetries. Therefore the points $\mathrm{X}$ and $\mathrm{Y}$, as well as $\mathrm{A}$ and $\mathrm{T}$ should always be read as shown in Fig. 1(b) and parametrized above. The BZ for side-centered lattices is only relevant for SGs with crystallo- graphic point group $m m 2$. The rotation axis is always chosen to be along the [001] direction, which requires the use of the site-centered lattice for SGs 38-41. As convention for this paper, we rotate the BZ in Fig. 1(b) around the $k_{y}$ axis. This way, $\Gamma-X$ and Z-A always correspond to the rotation axis $\left(0,0, k_{z}\right)$ and $\left(\frac{1}{2}, 0, k_{z}\right)$, whereas the lines $\Gamma-Y$ and Z-T are to be understood as the lines perpendicular to it.

In face-centered lattices, we also find four TRIMs with maximal symmetry, again $\Gamma, \mathrm{Z}, \mathrm{Y}$, and $\mathrm{T}$. The remaining four TRIMs are symmetry-related copies of L and have only the identity in their site symmetry group. Depending on the relative length of the conventional lattice constants $a, b$ and $c$, there are several distinct shapes the BZ can take, of which we show the one for $\frac{1}{a^{2}}=\frac{1}{b^{2}}+\frac{1}{c^{2}}$ in Fig. 1(c). Again we introduce additional labels to allow for a unique declaration of directions, $\Gamma-\mathrm{X}=(u, 0,0)$ connecting $\Gamma$ and $\mathrm{T}, \mathrm{Y}-\mathrm{T}=$ $(0,2 \pi, w) \sim(2 \pi, 0, w)$ and $\mathrm{Z}-\mathrm{A}=(u, 0,2 \pi)$ connecting $\mathrm{Z}$ with $Y$. The results presented in this paper do not depend on the actual shape of the BZ, as long as the correct orientation is chosen and high-symmetry lines should always be understood as defined above and visualized in the exemplary BZ.

The last lattice type found for the orthorhombic SGs has a body-centered conventional cell. Only $\Gamma$ and $\mathrm{Z}=$ $(0,0,2 \pi) \sim(2 \pi, 0,0) \sim(0,2 \pi, 0)$ have maximal symmetry. The other TRIMs S, R, and T have the symmetry of the first, second and third symmetry axis, respectively, and exist in two symmetry related copies. Additionally, there is the high-symmetry point $\mathrm{W}$, which is invariant under all rotations, but not under $\mathcal{T}$, see Fig. 1(d). In all cases, we specify highsymmetry planes through parametrization in the Cartesian coordinates, e.g., $k_{z}=\pi$.

\section{SCREENING FOR CANDIDATE MATERIALS}

For 14 of the orthorhombic SGs (SGs 18, 19, 24, 30, 33, 34, $43,52,56,57,60,61$, and 62) that exhibit some of the most notable symmetry-enforced features listed in Tables I-IV, we perform a computationally-assisted screening of the Materials Project (MP) database [30,31] for real material examples. We restrict our search to phases which have corresponding entries in the Inorganic Crystal Structure Database (ICSD, FIZ Karlsruhe) - essentially, the space of known, ordered, inorganic crystals. The distribution of these phases by SG is depicted in Fig. 2(a), with incidence varying by three orders of magnitude. 
(a)

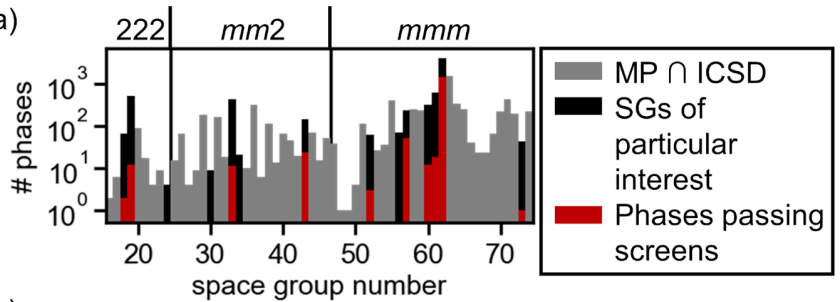

(b)

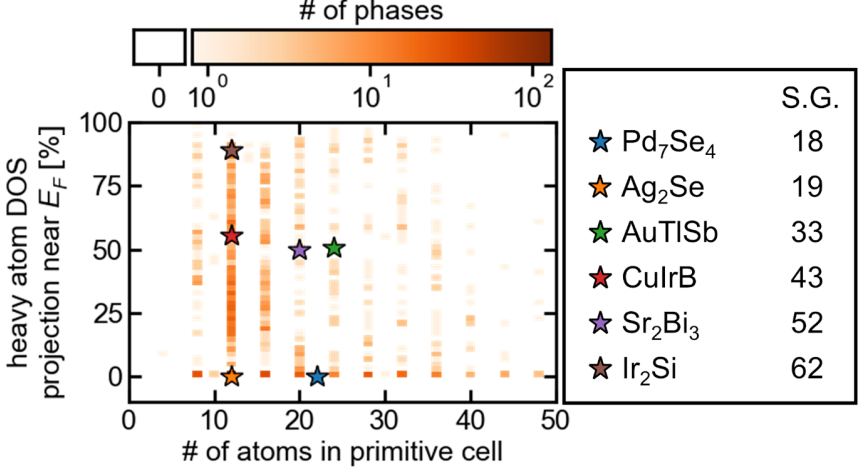

FIG. 2. (a) Distribution of ordered, orthorhombic phases in the Materials Project and ICSD by SG number (MP $\cap$ ICSD, $N=$ 12,292). 14 SGs of particular interest are indicated, as well as the number of phases in these SGs passing the automated screening criteria described in the text. The crystallographic point groups are indicated above the plot. (b) 2D histogram of phases passing the automated screening criteria by fraction of the projected DOS near the Fermi energy contributed by heavy atoms (see Ref. [49]) and number of atoms in the primitive unit cell $(N=1259)$. We note that all the phases that passed the screening have an even number of atoms in the primitive cell, since all orthorhombic SGs with nonsymmorphic symmetries have even Wyckoff multiplicities. The specific example materials discussed in the text are highlighted by the stars.

We apply several preliminary screens on thermodynamic stability (formation energy $\leqslant 50 \mathrm{meV}^{-1}$ atom $^{-1}$ off the convex hull), structural complexity $(\leqslant 50$ atoms in the primitive unit cell, as larger unit cells tend to have more crowded and flatter bands), and electronic band gap $(\leqslant 0.1 \mathrm{eV}$ by the generalized gradient approximation). We note that many insulating crystals have the desired SG symmetries, but their more localized bands tend to diminish the importance of the symmetryenforced crossings, for instance by making crossings poorly isolated in momentum and energy from other states. In addition, many insulating crystals may be difficult to degenerately dope to position the Fermi energy near crossings of interest. As a result, we focus here on metals, semimetals, and nearly gapless semiconductors. Together, these preliminary screens narrow the search to $\sim 1540$ phases across these 14 SGs (ranging from zero phases for SGs 24, 30, 34, and 56 to $~ 1400$ phases for SG 62). To isolate cases of both strong and weak SOC near the Fermi level, we use the electronic densities of states (DOS) computed without SOC in the MP database (present for $\sim 1260$ of the remaining phases, or $82 \%$ ) to assess what fraction of the orbital character is due to heavy atoms. Specifically, we define a heavy atom DOS fraction near $E_{F}$ as the integral of the DOS projection onto orbitals from atoms indium or heavier $(Z \geqslant 49)$ divided by the integral of the DOS projection onto all orbitals, evaluated on the energy window
$\left[E_{F}-1 \mathrm{eV}, E_{F}+1 \mathrm{eV}\right]$. Typical of plane-wave codes, the total projections onto these atom-centered spherical harmonics are not quantitative due to the arbitrary definition of the atomic radii, but this ratio is nonetheless indicative of the contribution from heavy atoms. The phases passing the screening are given in Ref. [49], the distribution of these phases by heavy atom DOS projection near $E_{F}$ and number of atoms in the primitive cell is given in Fig. 2(b). Since all SGs of interest contain at least one nonsymmorphic symmetry, the lowest multiplicity for a Wyckoff position is two or four. Therefore there are no phases with an odd number of atoms in the unit cell among the results. Several example materials are annotated which will be discussed subsequently. As a last step to identify high-quality material examples, we examine the experimental literature to assess synthesizability, evaluate whether the computed GGA band structure is consistent with experimental observations (e.g., color and luster, resistivity, magnetization) and confirm that the crystal structure model in the desired SG has not been superceded by one of a different symmetry in subsequent investigations.

For these example materials, we performed electronic structure calculations using the VASP code [50,51], which implements the Kohn-Sham formulation of density functional theory (DFT) using a plane wave basis set and the projector augmented wave formalism [52,53]. All calculations were performed with the PBE exchange-correlation functional [54], and optimized structures from the MP database were used directly. The band structures calculated along high-symmetry paths are presented in Appendix A. Surface states were calculated via a Wannier model using WANNIER90 [55] and WANNIERTOOLS [56].

\section{RHOMBIC DISPHENOIDAL: SG 16-SG 24}

The chiral SGs in the orthorhombic crystal system, SGs 16 to 24 , have the crystallographic point group $D_{2}$, or 222 in the Hermann-Mauguin notation, consisting of three perpendicular twofold rotations. Since neither mirror planes nor inversion are present, these SGs are chiral.

Symmetry-enforced features are (movable) Weyl points, nodal planes and fourfold degenerate points with a topological charge of $|\mathcal{C}|=2$, called fourfold double Weyl points [19].

\section{A. Weyl Points at high-symmetry points}

Time-reversal symmetry $\mathcal{T}$ squares to a $2 \pi$ rotation and therefore -1 in spinful representations. Kramers theorem implies twofold degeneracies at all TRIMs in spinful band structures. Without further restrictions from additional symmetries, these degeneracies are Weyl points with linear dispersion and a chirality $\mathcal{C}= \pm 1[24,25,57]$. In the chiral orthorhombic SGs 18-24 with strong SOC, every Kramers degeneracy at a TRIM that is not part of a nodal plane (see Sec. IV C below) is such a Kramers-Weyl point. They are listed in the column "Points" of Table II.

In spinless representations, $\mathcal{T}$ squares to the identity and Kramers theorem does not apply. Consequently, the bands at TRIMs are in general not degenerate. There is however one case of a symmetry enforced Weyl point at a high-symmetry point in SG 24. The symmetries in this body-centered SG are 
three orthogonal, off-centered rotations. While twofold rotations without translational components always commute, the resulting translation in the combination of two off-centered rotations depends on their order. For the generators of SG 24, we find, for example,

$$
\begin{aligned}
& 2_{100}\left(0,0, \frac{1}{2}\right) 2_{001}\left(0, \frac{1}{2}, 0\right) \\
& \quad=(-1)^{\zeta} t(0,-1,0) 2_{001}\left(0, \frac{1}{2}, 0\right) 2_{100}\left(0,0, \frac{1}{2}\right) .
\end{aligned}
$$

There are three points in the Brillouin zone with maximal symmetry, of which only $\mathrm{W}$ has translation eigenvalue -1 for the above translation by one lattice constant in the conventional cell. Therefore the spinless representations of the rotations $U_{2_{001}}$ and $U_{2_{100}}$ anticommute and must be at least two-dimensional [58,59]. For an eigenstate $| \pm\rangle$ of $U_{2_{001}}$ with eigenvalue \pm 1 , we find

$$
\begin{aligned}
U_{2001} U_{2_{100}}| \pm\rangle & =-U_{2_{100}} U_{2_{001}}| \pm\rangle \\
& =\mp U_{2_{100}}| \pm\rangle,
\end{aligned}
$$

which implies that for example $|+\rangle$ and $U_{2_{100}}|+\rangle \propto|-\rangle$ are two distinct states. Because the Hamiltonian commutes with $U_{2_{001}}$ and $U_{2_{100}}$, they will be degenerate. In comparison, SG 23 has no translational parts in its rotations and the translation eigenvalue of -1 is missing in the commutation relation. Instead, Eq. (4.2) holds for spinful representations. In both cases states with opposite rotation eigenvalues are paired at $\mathrm{W}$, the gap opens linear to lowest order in momentum and the point degeneracy carries a topological charge of $\mathcal{C}= \pm 1$ [25]. Notably, this Weyl point is enforced even in the absence of time-reversal symmetry.

If the spin degrees of freedom in $\mathrm{SU}(2)$-symmetric band structures in SG 24 are considered, the topological charges of the Weyl points at $\mathrm{W}$ in each spin space are identical and the total topological charge is $\mathcal{C}_{\uparrow}+\mathcal{C}_{\downarrow}= \pm 2$. Including SOC requires the use of spinful representations, where the above relation does not hold, due to an additional sign change from the spin part of the symmetries in the commutation relation. As we will show in the next chapter, there is a weaker requirement in that case for two separate Weyl points on a rotation axis connecting to W. As long as SOC is small, these two Weyl points remain close to $\mathrm{W}$, and the Chern number calculated on a surface enclosing the two Weyl points is not changed by SOC.

\section{B. Movable Weyl points on rotation axes}

A movable Weyl point is not fixed to a high-symmetry point. Such Weyl points are enforced when compatibility relations between representations at high-symmetry points and the axis connecting them requires an exchange of bands with different symmetry eigenvalues [60]. The resulting band crossing is therefore symmetry protected, but its position on the axis is not fixed, hence it is called movable.

Twofold screw rotations square to lattice translations in the direction of the rotation axis. Hence, their eigenvalues are the square roots of translation eigenvalues and therefore $k$-dependent. For example, $2_{001}(a, b, c)$ has eigenvalues

$$
U_{2001}| \pm\rangle= \pm \mathrm{i}^{\zeta} \exp \left(\mathrm{i} k_{z} c\right)| \pm\rangle
$$
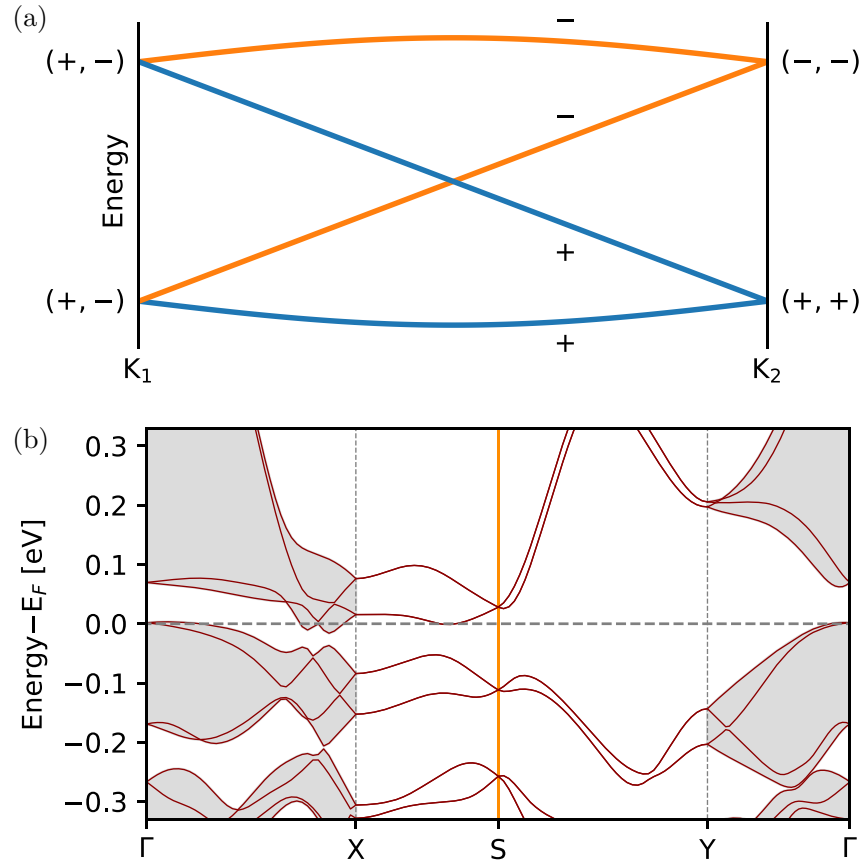

FIG. 3. (a) Scheme of an hourglass dispersion along a path between two degenerate points $K_{1}$ and $K_{2}$ along a twofold rotation axis or in a mirror plane. Colors indicate the sign of the symmetry eigenvalue of the twofold symmetry. (b) Band structure for $\mathrm{Pd}_{7} \mathrm{Se}_{4}$ in SG 18 with SOC showing hourglass states on $\Gamma$-X (less clearly at $\Gamma$-Y) colored in gray and fourfold double Weyl points at S, highlighted by the orange line.

where $c=\frac{1}{2}$ for a screw rotation. Along the rotation axis the eigenvalue of each band evolves smoothly with $k_{z}$, and a state can be labeled uniquely by the sign \pm according to this definition. Throughout the paper, we will use blue and orange in plots for states with positive and negative signs, respectively. Kramers partners at TRIMs have complex conjugate eigenvalues. For a TRIM on the axis invariant under the rotation with $k_{z}=0$, the spinful eigenvalues $\pm \mathrm{i}$ are paired. We label such a pair in terms of the signs $(+,-)$. At TRIMs with $k_{z}=\pi$ the eigenvalues are $\pm(-1)$ and identical eigenvalues are paired, $(+,+)$ or $(-,-)$. Connecting these pairs smoothly along the rotation axis requires four states in total and at least one band crossing in between the two TRIMs, leading to a so-called hourglass dispersion [61], see Fig. 3(a) for an illustration. Without further restrictions from other symmetries in the SG, such a band crossing is a Weyl point. The rotation axes on which these movable Weyl Points occur are listed in the column "Points" in Table II by specifying the rotation axis with the number of bands involved given in brackets. Movable Weyl points enforced by this mechanism are found in spinful band structures of the chiral SGs 17-20 on all screw axes.

In spinless systems, $\mathcal{T}$ squares to the identity and there are in general no Kramers degeneracies at TRIMs and no symmetry-enforced hourglass states. In the case of three screw rotations in SG 19 and 24, however, a movable crossing is still enforced by an extended compatibility relation, meaning the combined compatibility relations on all three rotation axes together with the group structure at their intersections [21]. 
(a)

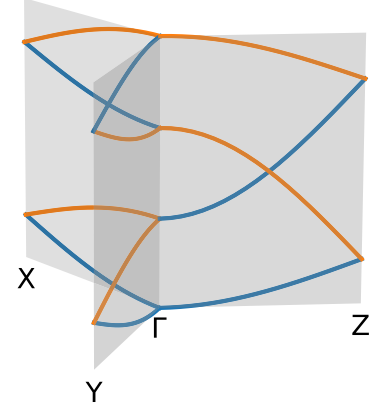

(b)

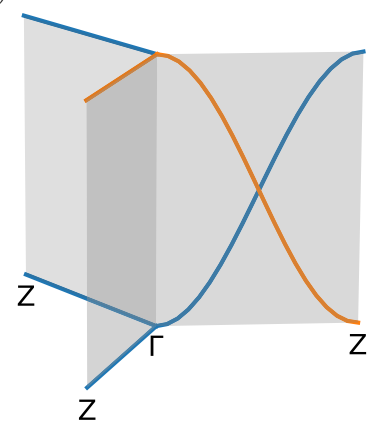

FIG. 4. Band crossings enforced by compatibility relations comprising three rotation axes. (a) Movable Weyl point in spinless band structures of SG 19 on one of the three rotation axes connecting to $\Gamma$. Blue (orange) colored bands correspond to bands labeled $+(-)$ according to the definition in Eq. (4.3). (b) Possible arrangement of spinless bands in SG 24 with rotation eigenvalue +1 in blue and -1 in orange of the corresponding axis. The product of eigenvalues is fixed to +1 at $\Gamma$ and -1 at Z, enforcing a movable Weyl point on one of the axes.

Starting with SG 19, we note that spinless representations have eigenvalues $\pm \mathrm{i}$ at the TRIMs $\mathrm{X}, \mathrm{Y}$, and $\mathrm{Z}$ according to Eq. (4.3) for $2_{100}, 2_{010}$, and $2_{001}$, respectively. There they are paired as $(+,-)$ by time-reversal symmetry. At $\Gamma$, all three rotation eigenvalues are \pm 1 and simultaneously good quantum numbers. We label a state $\left|s_{100}, s_{010}, s_{001}\right\rangle$ using the signs $s_{i}= \pm$ of all three rotations. The product of all three rotations result in a translation with eigenvalue 1 , and therefore the product of rotation eigenvalues \pm 1 must also be +1 . Consequently, only the combinations $|+,+,+\rangle,|+,-,-\rangle$ and permutations thereof are valid. Connecting these states smoothly to the pairs $(+,-)$ at the other TRIM on each axis, i.e., $\mathrm{X}, \mathrm{Y}$, or $\mathrm{Z}$, requires all four possible combinations to be present at $\Gamma$ and at least one crossing on one of the rotation axes [62], see Fig. 4(a). Since crossings of bands with different eigenvalues are symmetry protected, exchanging the bands can only move the crossing to another axis, but not eliminate it, and additional crossings can only be introduced pairwise. These movable Weyl points on one of the three rotation axes are indicated in the column "Points" by $\Gamma-[X \underline{\vee} Y \underline{V}]$. Here, the symbol $\underline{\vee}$ stands for "exclusive or" between the different high-symmetry points, as only one crossing on one of the three rotation axes is symmetry enforced. In the absence of additional accidental crossings, which might appear on any of the rotation axes, SG 19 has only three pointlike degeneracies for $4 n+2$ filled bands and the topological charge from the two symmetry-related copies of the movable point is compensated by the fourfold double Weyl point at R. See Sec. IV D below.

Next, we consider SG 24, which has a body-centered BZ where three rotation axes connect $\Gamma$ to Z, see Fig. 1(d). Both TRIMs $\Gamma$ and $Z$ are nondegenerate in spinless representations of SG 24. Because of the translational parts of the rotations, the product of all three rotations results in a lattice translation,

$$
2_{100}\left(0,0, \frac{1}{2}\right) 2_{010}\left(\frac{1}{2}, 0,0\right) 2_{100}\left(0, \frac{1}{2}, 0\right)=t\left(\frac{1}{2},-\frac{1}{2}, \frac{1}{2}\right) .
$$

At $\Gamma$, the translation eigenvalue is 1 and the product of all three rotation eigenvalues \pm 1 has to be positive, as before. Note that the rotation eigenvalues of these off-centered rotations are not $k$-dependent, cf. Eq. (4.3) with $c=0$. At Z on the other hand, the eigenvalue of the translation is -1 , therefore the only valid combinations are $|-,-,-\rangle$ and $|+,+,-\rangle$ and permutations thereof. The two different requirements can only be fulfilled simultaneously by two bands exchanging on at least one rotation axis. An example for states $|+,+,+\rangle$ and $|+,-,-\rangle$ at $\Gamma$ and $|+,-,+\rangle$ and $|-,+,+\rangle$ at $\mathrm{Z}$ is shown in Fig. 4(b). While the band representation at $\Gamma$ and $\mathrm{Z}$ in a real material is induced from the orbital present at a certain Wyckoff position [16] and the ordering of states in energy is dependent on material parameters, the restriction on the dispersion is always the same as in the example. The charge of this movable Weyl point is, in the absence of accidental crossings, compensated by the pinned charge at $\mathrm{W}$, which also appears in two symmetry-related copies.

The above relations leading to the movable crossing hold for spinless representations only. However, treating weak SOC as a perturbation to a spin-degenerate band structure will not remove the Weyl points. As seen from a closed surface surrounding the band crossing, the Chern number evaluated on this surface is not changed by SOC, as long as the gap on the surface remains open. The two copies of the Weyl point might split and leave the rotation axis, but only a large perturbation can remove them from the band structure altogether.

In addition, we find in spinful bands of SG 24 a movable Weyl point on one of the three axes W-R, W-S, or W-T. The compatibility relations require the same connectivity as shown in Fig. 4(a), just with inverted eigenvalues, because all three rotation eigenvalues at $\mathrm{W}$ multiplied equal to $-\mathrm{i}$. If SOC vanishes exactly, the Weyl point in each spin subspace is pinned to $\mathrm{W}$, as has been shown above. With increasing SOC, they can split up and move onto an axis, where they are still related by symmetry.

\section{Material example $\mathrm{Pd}_{7} \mathrm{Se}_{4}$}

An example of a movable Weyl point with hourglass dispersion is found in $\mathrm{Pd}_{7} \mathrm{Se}_{4}$, which crystallizes in SG 18 [63], exhibiting metallic conductivity [64]. Single crystals large enough for diffraction were obtained by annealing the product obtained from the melt [63], but larger crystals may be challenging as it is incongruently melting with a very small exposed liquidus (e.g. for flux growth) [65]. The bands of $\mathrm{Pd}_{7} \mathrm{Se}_{4}$ are shown in Fig. 3(b) and exhibit, as expected, an hourglass structure on the axes $\Gamma-\mathrm{X}$ and $\Gamma-\mathrm{Y}$. The full band structure along high-symmetry paths is shown in Fig. 12(a). As we will discuss in Sec. IVD the compound also hosts fourfold double Weyl points.

\section{Topological nodal planes}

The combination of time-reversal symmetry and a twofold screw rotation leads to twofold degeneracies on a plane. These so called nodal planes can contribute to the band topology $[66,67]$. After discussing the mechanism forming these nodal planes, we identify cases where the nodal planes are enforced to be topologically nontrivial. 
The combined symmetry of a screw rotation and timereversal symmetry is antiunitary and squares to a full lattice translation along the rotation. The action on a point $\mathbf{k}$ in the $\mathrm{BZ}$ is similar to a mirror symmetry with its invariant plane orthogonal to the rotation axis. For example, $2_{001}\left(0,0, \frac{1}{2}\right) \mathcal{T}$ squares to $t(0,0,1)$ and transforms a point $\mathbf{k}$ according to $\left(k_{x}, k_{y}, k_{z}\right) \rightarrow\left(k_{x}, k_{y},-k_{z}\right)$. In the BZs of primitive and basecentered lattices, the plane $k_{z}=\pi$ is equivalent to $k_{z}=-\pi$ up to a reciprocal lattice translation and the eigenvalue of the translation is $\mathrm{e}^{\mathrm{i} k_{z}}=-1$. Any antiunitary symmetry squaring to -1 enforces degeneracies on invariant momenta through an extended Kramers theorem [19]. Consequently, the whole plane $k_{z}=\pi$ is twofold degenerate. Note that this holds equally for spinless and spinful representations, since both time reversal and the rotation square to a $2 \pi$ rotation such that the eigenvalue of the latter contributes no overall sign. These nodal planes split into nondegenerate bands linear in the momentum component normal to the nodal plane. We list nodal planes in the fourth column of Tables I-III. Notably, in SG 18, two such nodal planes exist on perpendicular planes, called a nodal plane duo. In SG 19, there are three screw rotations and the resulting nodal plane trio encloses the whole BZ.

Like a pointlike degeneracy, a nodal plane can act as a source or sink of Berry curvature and carry a topological charge. In that case, we refer to it as a topological nodal plane. All nodal planes in chiral SGs can carry a topological charge.

For band structures with strong SOC in SG 19, this is not only a possibility, but required by symmetry for the nodal plane trio. In order to find the nodal charge, it is sufficient to analyze all Weyl points that close the same gap as the nodal plane trio. Because of the Nielsen-Ninomiya theorem [68], their chiralities sum up to the negative topological charge of the nodal plane trio. In each elementary band representation, there is a Kramers-Weyl point at $\Gamma$ with a chirality of $\pm 1[27,28]$. Every additional Weyl point can only be at a $k$-point with a star of order 2,4 , or 8 , because all other points of maximal symmetry reside within the nodal planes. Because the different points of a star are related by time-reversal symmetry or rotations, the Weyl points have the same chirality. In total, we find

$$
\mathcal{C}_{\text {total }}= \pm 1+2 n \neq 0 \quad n \in \mathbb{Z} .
$$

Therefore the topological charge of the nodal plane trio has to be odd and $\left|\mathcal{C}_{\mathrm{NP}}\right| \geqslant 1$ [69].

\section{Fourfold double Weyl points}

Fourfold double Weyl points are fourfold degenerate points with a Chern number of $\mathcal{C}= \pm 2$ [19] and linear dispersion at lowest order in momentum. Their topology is equivalent to two superimposed Weyl points with equal Chern number and they have therefore also been called double-spin-1/2 Weyl points [70]. They should not be confused with the twofold degenerate double Weyl points with chirality $\mathcal{C}= \pm 2$, which can be stabilized only by higher order rotations [24] and have quadratic dispersion perpendicular to the rotation axis. An example of the dispersion in the proximity of a fourfold double Weyl point in $\mathrm{Pd}_{7} \mathrm{Se}_{4}$ is shown in Fig. 5 .

Two different mechanisms need to be at play simultaneously to enforce such a feature. First, the representations

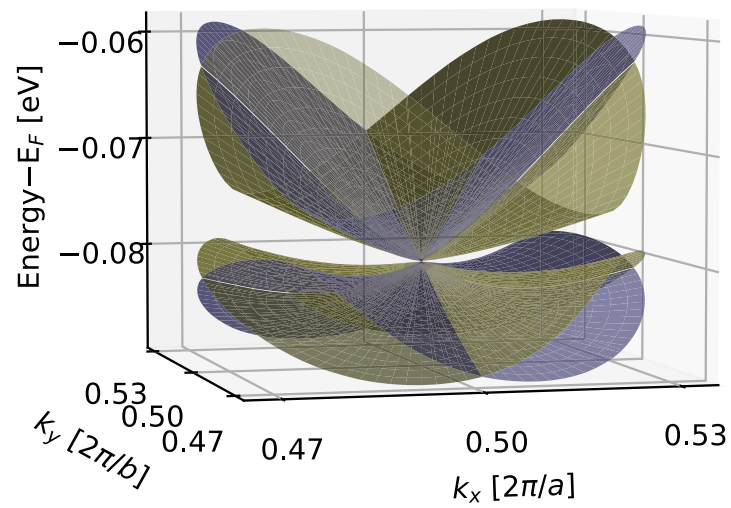

FIG. 5. Dispersion of the fourfold double Weyl point in $\mathrm{Pd}_{7} \mathrm{Se}_{4}$ within the $k_{z}=0$ plane around $\mathrm{S}=(\pi, \pi, 0)$. The colors indicate the two Weyl cones, related by the rotation $2_{100}$ or $2_{010}$. The twofold degenerate lines along $\left(k_{x}, \pi, 0\right)$ and $\left(\pi, k_{y}, 0\right)$ are part of nodal planes.

of the three twofold rotations at a TRIM need to anticommute and secondly, their eigenvalues must be real, such that time-reversal symmetry $\mathcal{T}$ relates identical eigenvalues. In orthorhombic SGs they are found in band structures with strong SOC in SG 18 at the TRIMs S and R, and in SG 19 at the TRIMs S, T, and U.

We illustrate the mechanism for $\mathrm{S}$ in SG 18, where the symmetries $\mathcal{T}, 2_{100}\left(\frac{1}{2}, \frac{1}{2}, 0\right)$, and $2_{010}\left(\frac{1}{2}, \frac{1}{2}, 0\right)$ are present at all TRIMs. A direct calculation shows the relation

$$
\begin{aligned}
& 2_{100}\left(\frac{1}{2}, \frac{1}{2}, 0\right) 2_{010}\left(\frac{1}{2}, \frac{1}{2}, 0\right) \\
& \quad=(-1)^{\zeta} t(1,-1,0) 2_{010}\left(\frac{1}{2}, \frac{1}{2}, 0\right) 2_{100}\left(\frac{1}{2}, \frac{1}{2}, 0\right)
\end{aligned}
$$

and at the TRIMs $\mathrm{S}$ and $\mathrm{R}$ we find therefore $U_{2_{100}} U_{2010}=$ $-U_{2_{010}} U_{2_{100}}$ in spinful representations, since there the translation eigenvalue is 1 for $t(1,-1,0)$. Applying $U_{2_{010}}$ to a state with a positive $2_{100}$ eigenvalue results in a new state with negative eigenvalue and vice versa. This requirement alone leads to a Weyl point with chirality $|\mathcal{C}|=1$ [25]. Because $2_{100}$ is a screw rotation, the eigenvalues at $\mathrm{R}$ and $\mathrm{S}$ are $\pm \mathrm{ie}^{\mathrm{i} k_{x} / 2}=\mp 1$. Kramers partners therefore have the same symmetry eigenvalue. In terms of 2100 -eigenvalues, we therefore find the quadruple degeneracy $(+,+,-,-)$. The chiralities of both time-reversal related copies are identical and add up to \pm 2 .

The dispersion around a fourfold double Weyl point is linear to first order [70,71]. Because of the necessary presence of time-reversal symmetry and screw rotation, all fourfold double Weyl points reside at intersections of nodal planes. Within these planes, the states remain twofold degenerate, otherwise they will split into four nondegenerate bands, see Fig. 5 for the dispersion in the plane $k_{z}=0$ of a fourfold double Weyl point in $\mathrm{Pd}_{7} \mathrm{Se}_{4}$.

In spinless band structures there is one case of a symmetry enforced fourfold double Weyl point at the TRIM R in SG 19 [62]. The principle remains the same, with the difference that the commutation relation for the screw rotations reads

$$
\begin{aligned}
& 2_{100}\left(\frac{1}{2}, \frac{1}{2}, 0\right) 2_{010}\left(0, \frac{1}{2}, \frac{1}{2}\right) \\
& \quad=(-1)^{\zeta} t(1,-1,-1) 2_{010}\left(0, \frac{1}{2}, \frac{1}{2}\right) 2_{100}\left(\frac{1}{2}, \frac{1}{2}, 0\right) .
\end{aligned}
$$


(a)

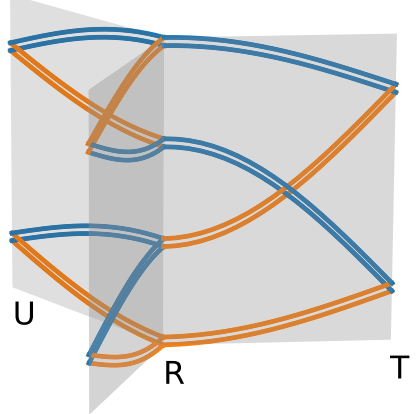

S

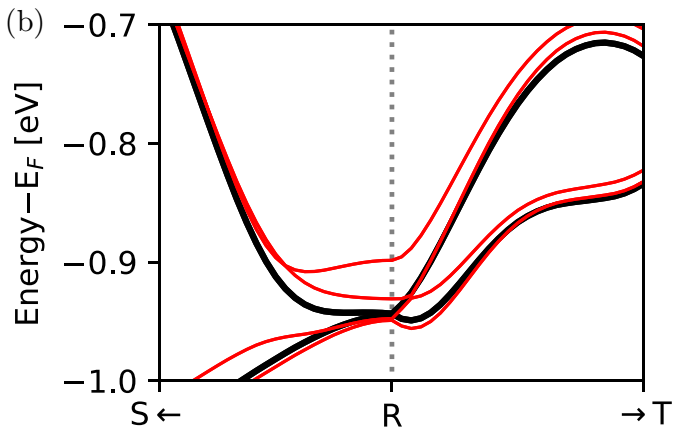

FIG. 6. (a) Compatibility relations at R in SG 19 for spinful bands. Colors indicate the sign of the rotation eigenvalue according to Eq. (4.3). Note that on the paths shown all bands are twofold degenerate with identical rotation eigenvalues paired. The figure adapted from Ref. [21]. (b) Fourfold double Weyl point in $\mathrm{Ag}_{2} \mathrm{Se}$ without SOC at R (black). Including SOC (red) moves the fourfold double Weyl point to the axis R-T. In both cases, bands are twofold degenerate.

In the case $\zeta=0$, the negative sign is provided by the translation eigenvalue of $t(1,-1,-1)$ at the TRIM R. Additionally, Kramers pairs are created by the combination of a screw rotation with time-reversal symmetry, e.g., $2_{001}\left(\frac{1}{2}, 0, \frac{1}{2}\right) \mathcal{T}$. Even though the eigenvalues of $U_{2100}$ and $U_{2010}$ at $\mathrm{R}$ are purely complex, identical eigenvalues are paired, since $U_{2_{001}}$ anticommutes with both representations, $U_{2_{100}}$ and $U_{2_{010}}$, and this cancels the sign change from complex conjugation. In the spinful case with nonzero SOC, there are fourfold double Weyl points at the TRIMs S, U, and T, and the fourfold double Weyl point at $\mathrm{R}$ moves to one of the three axis S-R, U-R, and T-R [21]. This movable point is required to exist due to the compatibility relations of the band structures that must be satisfied on the three rotation axes simultaneously. Figure 6(a) shows an example of an arrangement of bands fulfilling the compatibility relations on the three rotation axes through $\mathrm{R}$. All three axes are part of nodal planes, therefore all bands are twofold degenerate. Each pair is formed by two bands with the same eigenvalue, as indicated by the double lines in the same color.

\section{Material example: $\mathrm{Ag}_{2} \mathrm{Se}$}

We have identified $\mathrm{Ag}_{2}$ Se in SG 19 as an example of a material with fourfold double Weyl points and nodal plane trios. $\mathrm{Ag}_{2} \mathrm{Se}$ is a narrow gap semiconductor whose room temperature polymorph crystallizes in SG 19 [72]. As with the corresponding sulfide and telluride, it has been examined for its thermoelectric properties and fast ion conduction at high temperatures. In the vicinity of $\mathrm{R}$, the splitting of bands in $\mathrm{Ag}_{2} \mathrm{Se}$ is small with respect to the bandwidth. By comparison of DFT band structures without and with SOC, the connection between the pinned fourfold double Weyl point in the spinless case and how it splits into two movable ones upon including SOC is demonstrated in Fig. 6(b). The black lines show bands excluding contributions from SOC in which case there is a fourfold degeneracy pinned at R. Note that all bands are twofold degenerate because they are part of nodal planes. Taking SOC into account removes the spin degeneracy, but the twofold degeneracy in the nodal planes remains. There are now two fourfold double Weyl points at the axis R-T, related by time-reversal symmetry. Thus, the total topological charge is conserved. The band structure along the full high-symmetry path is shown in Fig. 12(b).

\section{RHOMBIC PYRAMIDAL: SG 25-SG 46}

SGs 25 to 46 have the crystallographic point group $C_{2 v}$ $(m m 2)$, consisting of a twofold rotation in the [001] direction and two mirror planes with normal along [100] and [010]. Because of the presence of mirror symmetries, these SGs are not chiral, but they are polar in [001] direction.

\section{A. Weyl points}

There cannot exist any point degeneracies with nonzero Chern number in mirror planes, since the Berry curvature transforms as a pseudovector under reflections and the contributions to a closed surface integral on either side of a mirror plane cancel. In base-, side- and body-centered lattices however, not all TRIMs reside in mirror planes. In band structures with strong SOC, these TRIMs host Kramers-Weyl points whenever they are not in a nodal plane. The difference to chiral SGs lies in the relative sign of Chern numbers for symmetry-related Weyl points. If two Weyl points are mapped onto each other by a mirror symmetry, they have opposite chirality. Consequently, the nodal planes $k_{z}=\pi$ in SGs 26, 29, 31, 33 and 36 cannot be topological, because the chiralities of all Weyl points in the interior of the BZ will always add up to zero.

Band structures in SGs 36 and 45 with SOC have additionally movable Weyl points. In the base centered lattice of SG 36 , the axis R-S has only the screw rotation and translations in its little group and an hourglass dispersion along this axis is enforced by the same mechanism as in the chiral SGs, see Sec. IV B. Because this axis is the only place for Weyl points, SG 36 has only two symmetry-enforced Weyl points at $S$ for an odd number of filled bands $n$ within a group of bands, while $\mathrm{R}$ is part of a nodal plane. For $n$ even, there are four Weyl points in total on the two axes S-R. Note that while there can be additional accidental Weyl points, it is in principle possible to have only two Weyl points in materials with SG 36. The existence of additional accidental Weyl points depends on the particular band dispersions and needs to be checked for a given material by inspection of the DFT bands. 
The rotation axis T-W in SG 45 gets an hourglass dispersion from a slightly different mechanism. The twofold rotation is not a screw rotation, therefore the eigenvalues are $\pm \mathrm{i}$ on the whole axis. However, $\mathrm{W}$ is not a TRIM, instead it is invariant under the combined symmetry $M_{010}\left(\frac{1}{2}, \frac{1}{2}, 0\right) \mathcal{T}$. This symmetry is antiunitary as well and enforces Kramers pairs on the axis R-W, equivalently $M_{100}\left(\frac{1}{2}, \frac{1}{2}, 0\right) \mathcal{T}$ with the invariant axis S-W. Because the representation for the mirror symmetry anticommutes with the one for the rotation at $\mathrm{W}$, the sign change from complex conjugation is compensated and identical eigenvalues are paired, $(+,+)$ and $(-,-)$, as opposed to $(+,-)$ at $\mathrm{T}$. Connecting these pairs leads again to the hourglass dispersion shown in Fig. 3(a).

In the spinful case, SGs 36,45 , and 46 have only two Weyl points connecting pairs of bands. This is the lowest possible number of Weyl points in any band structure and makes these SGs interesting candidates for investigating the effects from such topological point degeneracies. There are however additional degeneracies closing the gap along lines and, in the case of SG 36, a nodal plane. Additionally it can be noted, that SGs 36 and 45 have four Weyl points connecting pairs of bands. Note that there are two nonequivalent copies of S-R in the BZ, related by a mirror symmetry. Therefore the topological charges of the two Weyl points will cancel in all cases. Four Weyl points at TRIM can also be found in SGs 35 and 37 at R and S, and in the face-centered lattices of this group, namely in SG 42 and 43 at the TRIM L. A material example for the movable Weyl points in SG 36 is $\mathrm{AsPb}_{2} \mathrm{Pd}_{3}$, where the enforced Weyl points occur within about $300 \mathrm{meV}$ above and below the Fermi energy [73].

\section{B. Pinned nodal lines}

As shown above, there are no Kramers-Weyl points in mirror planes. However, Kramers theorem still holds and spinful band structures at TRIMs must be at least twofold degenerate. These degeneracies are always part of nodal lines and in most cases, these nodal lines are pinned to high-symmetry lines. Nodal lines split linearly into nondegenerate bands and are characterized by a $\mathbb{Z}_{2}$ invariant, namely, a Berry phase of $\pi$ picked up by any path encircling the nodal line. Pinned nodal lines can be readily identified from tables of irreducible representations for the little groups of the high-symmetry line, e.g., using the Bilbao Crystallographic Server [16]. For completeness and quick reference, we included them in Tables I to IV in the column "Lines."

For example, the rotation axis $\Gamma-\mathrm{Z}$ with little group $\mathrm{mm} 2$ is always twofold degenerate in spinful band structures, even in the absence of $\mathcal{T}$. Because the spin parts of the symmetries anticommute, all irreducible representations must be at least two-dimensional and eigenvalues of all symmetries are paired as $(+,-)$.

Another mechanism for pinned nodal lines results from the combination of glide mirror symmetries with time-reversal symmetry. A glide mirror symmetry has a translational part $\frac{1}{2} \mathbf{t}_{\perp}$ within the mirror plane. In double groups, mirror symmetries square to a $2 \pi$ rotation and in the case of a glide mirror symmetry an additional lattice translation $\mathbf{t}_{\perp}$ within the mirror plane. The eigenvalues are therefore of the form

$$
U_{M}| \pm\rangle= \pm \mathrm{i}^{\zeta} \exp \left(\frac{\mathrm{i}}{2} \mathbf{k} \cdot \mathbf{t}_{\perp}\right)| \pm\rangle .
$$

The combination of the glide mirror with $\mathcal{T}$ acts like a rotation in $k$ space with its invariant axis parallel to the mirror normal and running through TRIMs in the mirror plane, see the green lines in Fig. 1. On an axis through a TRIM $\mathbf{K}$ with $\mathbf{K} \cdot \mathbf{t}_{\perp}= \pm \pi$, the combined symmetry squares to -1 and enforces Kramers pairs by the extended Kramers theorem. For example, the glide mirror symmetry $M_{010}\left(\frac{1}{2}, 0,0\right)$ in SG 28 combined with $\mathcal{T}$ enforces the lines X-S and T-R to be twofold degenerate.

\section{Movable nodal lines}

The $k$-dependent eigenvalues of glide mirror symmetries defined in Eq. (5.1) lead to the possibility of pairing identical and opposite eigenvalues at different points in the same mirror plane. This leads to an hourglass dispersion as shown in Fig. 3(a) for any path in the mirror plane between these points [60]. The movable crossings on all these paths form a nodal line, which might either form a closed loop around one of the points or extend through the BZ. For example, in SG 28 with strong SOC, the glide mirror symmetry $M_{010}\left(\frac{1}{2}, 0,0\right)$ has eigenvalues $\pm \mathrm{i}$ at $\Gamma$-Z and Y-T, paired on the whole line, and eigenvalues \pm 1 for $k_{x}=\pi$, forming Kramers pairs $(+,+)$ and $(-,-)$ at $\mathrm{X}, \mathrm{U}, \mathrm{S}$ and $\mathrm{R}$. The movable nodal line in the $k_{y}=0$ plane therefore runs between $\Gamma-\mathrm{Z}$ and $\mathrm{X}$ or $\mathrm{U}$. A movable line is given in Tables I and II in terms of a tuple, e.g., $(\Gamma-Z ; R, U)$, where the entries left of the semicolon are the degeneracies with opposite eigenvalue pairing and the entries on the right the points where identical eigenvalues are paired. The mirror plane can be inferred from the high-symmetry points and lines in the bracket, otherwise it is specified explicitly. If a point appears left and right of the semicolon, there is a fourfold degeneracy with eigenvalues $(+,+,-,-)$ and the point is written in italic font. See Sec. VF below for a discussion of fourfold degenerate points.

Monolayer GaTeI can be considered as a subset of the 3D SG 31 with SOC, which exhibits hourglass nodal lines that correspond to $(\Gamma-\mathrm{Z}-\mathrm{U} ; \mathrm{X})$ in our notation [74]. While this feature is at about $1.2 \mathrm{eV}$ above the Fermi energy, it has been suggested that due to the two-dimensional geometry it may be possible to introduce a significant number of charge carriers by electrostatic gating.

\section{Nodal chain}

When two mirror planes with movable nodal lines intersect, it can be required that the nodal lines touch on the intersection and form a nodal chain [20]. TRIMs at the intersection of two glide mirror symmetries might have different eigenvalue pairing, i.e., $(+,-)$ for one mirror symmetry and $(+,+)$ for the other. If the pairing is different for both mirror symmetries at another TRIM on the same intersection, the requirements of an hourglass dispersion need to be fulfilled for both mirror symmetries simultaneously. Because the total number of crossings must be odd, the two movable nodal lines have to meet at one point on the axis. In case the TRIMs on the high-symmetry axis have opposite eigenvalue pairing as mentioned above, the two loops form a so called nodal chain, found in SGs 30, 34, and 43 with SOC [20]. In Table II, they can be identified by two entries for movable nodal lines with 
the two TRIMs changing sides with respect to the semicolon. For example, in the hourglass relations for SG 43, T and Y appear on each side once. In the $k_{x}=0$ plane, the relation reads $(\Gamma-Z-T ; Y)$ and $\mathrm{Y}$ has identical $M_{100}$ eigenvalues paired. On the other hand we find $\mathrm{Y}$ as part of the line $\mathrm{Z}-\mathrm{A}$ on the left in the relation for the $k_{y}=0$ plane, $(\Gamma-Z-\mathrm{A} ; \mathrm{T})$, and $\mathrm{T}$ has identical $M_{010}$ eigenvalues paired. On the line Y-T, both eigenvalues are good quantum numbers and both have to exchange as part of nodal lines. Consequently, the two nodal lines have to intersect and form a chain.

\section{Material example: $\mathrm{CuIrB}$}

Our search has identified CuIrB as an example of a material with nodal chains. CuIrB, obtained from the elements either by heating in quartz ampules or by arc-melting and annealing, crystallizes in SG 43 and exhibits metallic resistivity [75]. The nodal chain made from lines in the $k_{y}=0$ and $k_{x}=2 \pi$ planes are shown in Fig. 7(a) and the dispersion along the intersection of the mirror planes is presented in Fig. 7(b). The dispersion along the full high-symmetry path as defined in Fig. 13 is shown in Fig. 12(d).

\section{E. Almost movable lines}

There is one case of symmetry-enforced nodal lines not covered by the above discussion. The Kramers pairs at the TRIMs S and R in SG 44 and S in SG 46 have only a mirror symmetry in their little group and are not part of a pinned nodal line. The mirror eigenvalues are $\pm \mathrm{i}$ and $\mathcal{T}$ creates pairs $(+,-)$. In the vicinity of such a TRIM $\mathbf{K}$, a state at $\mathbf{K}+\delta$ in the mirror planes is mapped by $\mathcal{T}$ to a state with opposite eigenvalue at $\mathbf{K}-\delta$ with the same energy. Consequently, the two bands with opposite mirror eigenvalues have to exchange somewhere on any path within the mirror plane connecting these two points. The exchange of bands is protected by the different symmetry eigenvalues and the resulting nodal line is only pinned to the TRIM K. Otherwise it can be moved freely through the mirror plane and is therefore called an almost movable line [19]. Similar to movable nodal lines, we indicate these nodal lines also with a bracket containing the point of opposite eigenvalue pairing, but instead of a counterpart with equal eigenvalues we just leave a dash, e.g., $(\mathrm{S} ;-)$. The corresponding mirror symmetry and its invariant plane with the nodal line is always the one leaving the high-symmetry point invariant. Movable and almost movable nodal lines reside both in mirror planes and are made up of two opposite mirror eigenvalues. The splitting into nondegenerate bands is linear to lowest order and their topology is captured by a Berry phase of $\pi$.

\section{F. Fourfold degeneracies in mirror planes}

In Sec. VB, we have discussed two different types of pinned nodal lines. If they intersect a fourfold degeneracy follows. These fourfold degeneracies occur in two distinct types. The first type appears as a pointlike degeneracy in the gap between the nodal lines, whereas the second is crossed by an additional hourglass nodal line and a sphere in the BZ enclosing the fourfold degeneracy will be gapless for every number of filled bands. In both cases, bands disperse linearly
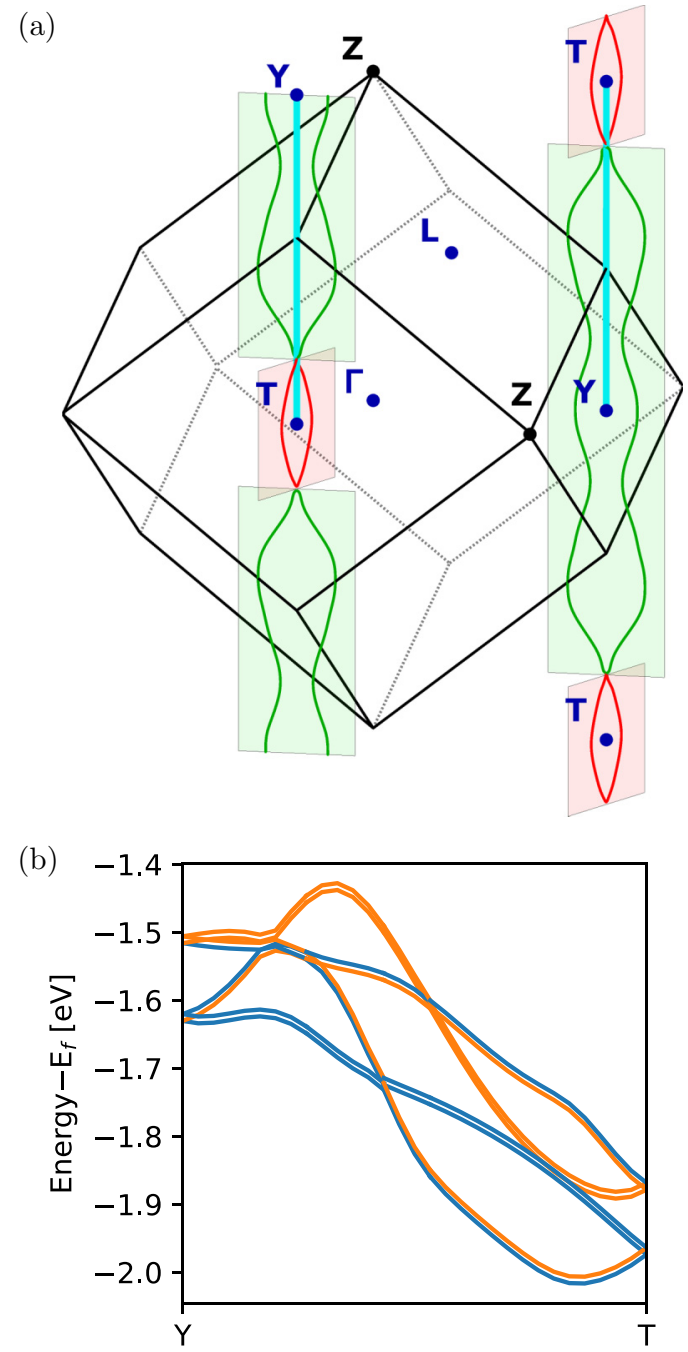

FIG. 7. (a) Nodal chain in CuIrB formed by the 10th and 11th valence band counted from the band gap at the Fermi energy. The green line is within the $k_{y}=0$ plane and encircles $\mathrm{Y}$, the red line is restricted to the other mirror plane $k_{x}=2 \pi$ and winds around T. (b) Dispersion on the intersection of both planes, shown in turquoise in (a). Blue and orange indicate positive and negative signs of the mirror eigenvalues according to Eq. (5.1), the upper color belongs to $M_{100}$ and the lower to $M_{010}$.

to lowest order in momentum in the vicinity of the fourfold degeneracy.

In the first type, the anticommutation relation of the two mirror symmetries $M_{1}$ and $M_{2}$ creates always eigenvalue pairs $(+,-)$. For the second type, at least one of the mirror symmetries, say $M_{1}$, needs to be nonsymmorphic with real eigenvalues at a TRIM. Time-reversal symmetry then pairs identical eigenvalues, requiring the fourfold degeneracy. The combined symmetry $M_{1} \mathcal{T}$ creates a twofold degeneracy along its invariant axis. Both pinned nodal lines are within the invariant plane of $M_{2}$. If its eigenvalues are also real at the fourfold degeneracy, then the combined antiunitary symmetry $M_{1} \mathcal{T}$ pairs opposite eigenvalues because of the anticommutation relation of mirror symmetries. In that case, the fourfold degeneracy is pointlike, but with vanishing Chern number. We list such cases in the column "Points" in Table II with the 

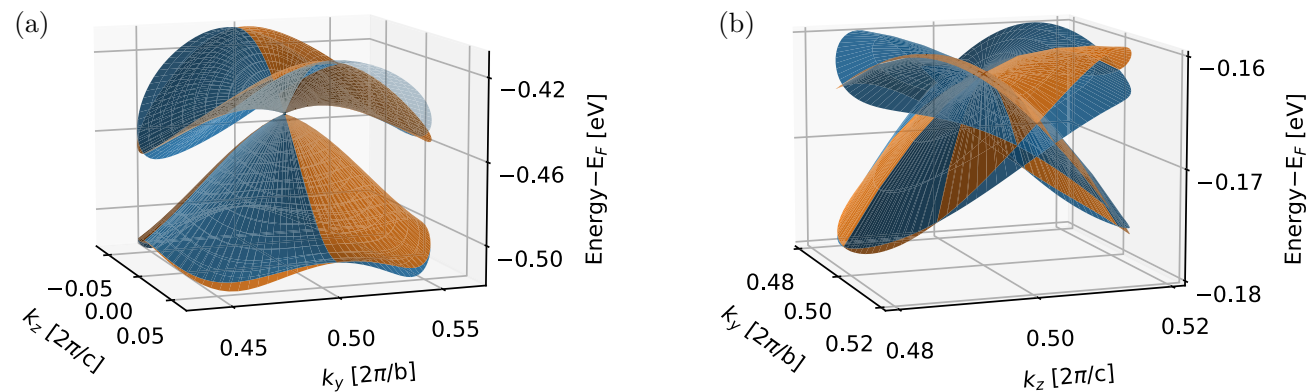

FIG. 8. Dispersion of the two different types of fourfold degenerate points in AuTlSb in the $k_{y}-k_{z}$-plane. Colors indicate the relative sign of $M_{100}$ eigenvalues. (a) Around $\mathrm{S}$ all lines are made of pairs $(+,-)$ and a pointlike degeneracy is formed. (b) At R, the nodal lines on $\mathrm{R}-\mathrm{Z}=(\pi, v, \pi)$ are paired $(+,+)$ and $(-,-)$, and an additional hourglass nodal line closes the gap, and thus the fourfold degenerate point is not a point crossing but part of several twofold degenerate nodal lines.

degeneracy given explicitly in brackets. An example is shown in Fig. 8(a) and the linearized low-energy Hamiltonian around such a point is given by Eq. (B9) in Appendix B 2.

The second possibility is complex eigenvalues for $M_{2}$. In that case, identical eigenvalues are paired by $M_{1} \mathcal{T}$ and there will be an hourglass nodal line in between the two pinned nodal lines [22]. Consequently, there is no gap, at which the fourfold degeneracy appears point like and we list the fourfold degeneracy in the columns "Lines" as an hourglass nodal line with the fourfold degenerate point on both sides of the semicolon and shown in italic, e.g., $(\mathrm{X}-U ; U-\mathrm{R})$. In this scenario, the two mirror symmetries must differ in their inplane translation and their product is always a screw rotation. See Fig. 8(b) for an example and Eq. (B13) for a linearized low-energy Hamiltonian.

\section{Material example: AuTlSb}

As an example, we present AuTlSb crystallizing in an anion-ordered pyrite-derivative structure in SG 33 and exhibiting metallic resistivity [76], see Fig. 12(c) for the band structure along the full high-symmetry path. Since the above considerations depend on pairing of $k$-dependent mirror eigenvalues, both types of fourfold degeneracies can occur in the same SG at different TRIMs. In SG 33, there are nodal lines at $\mathrm{S}-\mathrm{R}$ with $M_{100}\left(\frac{1}{2}, \frac{1}{2}, \frac{1}{2}\right)$-eigenvalues paired as $(+,-)$, because of the anticommutation relation of the two glide mirror symmetries. On the lines X-S and U-R, the combined antiunitary symmetry $M_{010}\left(\frac{1}{2}, \frac{1}{2}, 0\right) \mathcal{T}$ creates pairs $(+,-)$ and $(+,+) /(-,-)$, respectively. A fourfold point can therefore be found in the band structure of AuTlSb at S, see Fig. 8(a), whereas Fig. 8(b) shows the fourfold degeneracy with an hourglass nodal line at $\mathrm{R}$.

\section{RHOMBIC DIPYRAMIDAL: SG 47-SG 74}

The centrosymmetric SGs in the orthorhombic crystal system, SGs 47 to 74, have crystallographic point group $D_{2 h}$ $(\mathrm{mmm})$. The combination of inversion and time-reversal symmetry requires all states to be spin degenerate throughout the whole BZ via Kramers theorem. Any additional symmetryenforced degeneracy in spinful band structures is therefore a Dirac point or line. This overall twofold degeneracy already includes many of the degeneracies described above. For example, there are no nodal planes in centrosymmetric spinful band structures. Additionally, the Berry curvature must vanish identically at each point in the BZ and consequently no degeneracy might carry a nonzero Chern number.

\section{A. Dirac points at high-symmetry points}

We call a fourfold point degeneracy a Dirac point, if and only if its Chern number vanishes and it splits into twofold degenerate bands in every direction. The latter criteria is not always required in the literature for the definition of a Dirac point in a band structure [77], which would also include the fourfold point degeneracies in mirror planes discussed in Sec. VF. To lowest order in relative momentum, the splitting is also linear for all Dirac points stabilized by twofold symmetries. Dirac points pinned to TRIMs are also invariant under inversion [78]. A fourfold degeneracy is enforced, whenever the irreducible representation for inversion is two-dimensional and has eigenvalues \pm 1 . Their Kramers partner has to have the same eigenvalue and $\mathcal{T}$ enforces the fourfold degeneracy. As shown in Sec. IV A, the point W in the BZ of body-centered lattices hosts a Weyl point in SG 23 and SG 24 in spinless and spinful band structures, respectively, despite not being a TRIM. There are two possibilities of adding inversion to these two SGs. In spinless (spinful) band structures of SG 72 (SG 73), inversion combined with time-reversal symmetry doubles this degeneracy. The other possibility for the inversion center leads to SG 71 (SG 74). There, states at W remain only twofold degenerate and are part of a nodal line (the overall spin degeneracy). This leads to a single instance of a pinned Dirac point at a non-TRIM in SG 73. In the spinless case in SG 72, the fourfold degeneracy is not a Dirac point in the sense mentioned above, because bands are nondegenerate away from high-symmetry lines.

\section{B. Movable Dirac points}

In most cases, the overall Kramers degeneracy of band structures in centrosymmetric SGs prevents the hourglass dispersion from occurring on screw rotations, because rotation eigenvalues are paired as $(+,-)$ on the whole rotation axis.

There are, however, instances where the representation of $\mathcal{P} \mathcal{T}$ anticommutes with the screw rotation. More concretely, if the screw rotation is additionally off-centered with respect to the inversion center, exchanging the order of the two differs by a translation of a lattice vector [23]. This leads to a pairing 
of $(+,+)$ and $(-,-)$ on rotation axes where the eigenvalue of this translation contributes an additional minus sign. With Dirac points at the TRIMs, this leads again to the hourglass dispersion shown in Fig. 3(a). The only difference is, that all bands are twofold degenerate and eigenvalues appear twice. The movable crossing on the axis forms a Dirac point. We find these conditions to be met on at least one rotation axis in SGs $52,54,56$, and 60 . Following our previous notation, movable Dirac points are given in Table IV in terms of the two TRIMs on the rotation axis connected by a dash.

We find one additional movable Dirac point in SG 73 from an extended compatibility relation on the three rotation axis connecting $\mathrm{W}$ with $\mathrm{R}, \mathrm{S}$, and $\mathrm{T}$. In SU(2)-symmetric band structures, this corresponds to an eightfold degeneracy at W. With nonzero SOC, this degeneracy is lifted and there are two symmetry-related Dirac points on one of the rotation axes. Each TRIM has one rotation in its site symmetry group and eigenvalues are paired as $(+,+,-,-)$ and $(+,+)$ and $(-,-)$ on the axis. At $\mathrm{W}$, all three rotation eigenvalues are simultaneously present, but their overall sign is restricted to + . This can only be arranged with a minimum of one band crossing on one of the axes W-R, W-S, and W-T, equivalent to the requirement shown in Fig. 4(a).

\section{Movable and almost movable Weyl lines}

In spinless band structures of centrosymmetric SGs, glide mirror symmetries enforce movable nodal lines from an hourglass dispersion between high-symmetry lines and planes in the mirror plane [22]. The mechanism is the same as described in Sec. V C and we list them using the same notation in the column "Lines" in Table III. In all instances, the pinned and movable nodal lines cross in a common point, leading to a fourfold degeneracy as described in Sec. VF and shown in Fig. 8(b). Additionally, glide mirror symmetries might introduce almost movable lines whenever their eigenvalues at a TRIM are $\pm \mathrm{i}$, as described in Sec. V E.

\section{Movable Dirac lines}

In the spinful case, nodal lines can only be formed as a fourfold degeneracy, splitting linearly into twofold degenerate bands, called Dirac lines. Since the Berry phase is a $\mathbb{Z}_{2}$ invariant, its topological invariant needs to be evaluated in a spin subspace.

A movable Dirac line can only be enforced, if the Kramers pairs formed by $\mathcal{P} \mathcal{T}$ share the same mirror eigenvalue. This is the case, if and only if the glide mirror symmetry is additionally off-centered with respect to the inversion center. As an example we discuss SG 57, which has the glide mirror symmetries $M_{010}\left(0, \frac{1}{2}, \frac{1}{2}\right)$ and $M_{100}\left(0, \frac{1}{2}, 0\right)$, of which only the former is also off-centered. For a state $| \pm\rangle$ with $M_{010}$ eigenvalue $\pm \mathrm{i} \exp \left(\mathrm{i} \frac{k_{z}}{2}\right)$, we can calculate the eigenvalue for its Kramers partner $\mathcal{P} \mathcal{T}| \pm\rangle$ via the commutation relation of symmetries [79],

$$
M_{010}\left(0, \frac{1}{2}, \frac{1}{2}\right) \mathcal{P} \mathcal{T}=t(0,1,1) \mathcal{P} \mathcal{T} M_{010}\left(0, \frac{1}{2}, \frac{1}{2}\right),
$$

and therefore

$$
\begin{aligned}
U_{M_{010}} U_{\mathcal{P} \mathcal{T}} \mathcal{K}| \pm\rangle & =\mathrm{e}^{\mathrm{i}\left(k_{y}+k_{z}\right)} U_{\mathcal{P} \mathcal{T}} \mathcal{K}\left( \pm \mathrm{ie}^{\mathrm{i} \frac{k_{z}}{2}}\right)| \pm\rangle \\
& =\mp \mathrm{e}^{\mathrm{i} k_{y}} \mathrm{e}^{\mathrm{i} \frac{k_{z}}{2}} U_{\mathcal{P} \mathcal{T}} \mathcal{K}| \pm\rangle .
\end{aligned}
$$

From this it follows, that equal eigenvalues are paired in the plane $k_{y}=\pi$, where $\mathrm{e}^{\mathrm{i} k_{y}}=-1$. Additionally, there are further degeneracies at high-symmetry points in this plane even before taking time-reversal symmetry into account. On the axis Y-S, the representations of $M_{010}\left(0, \frac{1}{2}, \frac{1}{2}\right)$ and $M_{001}\left(0, \frac{1}{2}, \frac{1}{2}\right)$ anticommute. This readily creates eigenvalue pairs $(+,-)$, and together with the pairing from $\mathcal{P} \mathcal{T}$ a fourfold degeneracy $(+,+,-,-)$. At $\mathrm{R}$ and $\mathrm{T}$ however, the representation of $M_{010}\left(0, \frac{1}{2}, \frac{1}{2}\right)$ commutes with all other symmetries, but the representations of the other two mirror symmetries anticommute. This implies, that there are two orthogonal states for each $M_{010}\left(0, \frac{1}{2}, \frac{1}{2}\right)$ eigenvalue. They can be distinguished by their $M_{001}\left(0,0, \frac{1}{2}\right)$ eigenvalue, which is left invariant by $\mathcal{P} \mathcal{T}$, therefore the Kramers partners have identical eigenvalues for both mirror symmetries. This leads to a total of four degenerate states and the pairing in terms of $M_{010}\left(0, \frac{1}{2}, \frac{1}{2}\right)$ eigenvalues as defined in Eq. (6.2) is therefore $(+,+,+,+)$ or $(-,-,-,-)$. The movable nodal line formed by the exchange of bands $(+,+)$ and $(-,-)$ on any path connecting the differently paired states is fourfold degenerate and called a movable Dirac nodal line. In spinful centrosymmetric band structures, they are found in SGs 57, 60,61 , and 62. Following our previous notation for movable nodal lines, we indicate them in Table IV by a bracket containing the degeneracies with pairwise opposite eigenvalues on the left of the semicolon and the ones with four identical eigenvalues on the right. The example discussed above reads (S-Y;R,T).

In analogy to the nodal chains mentioned in Sec. V D, the two movable Dirac nodal lines in SG 60 both cross through the shared axis U-R and form a Dirac nodal chain with loops in the $k_{x}=\pi$ and $k_{z}=\pi$ planes. These Dirac nodal chains occur, for example, in the band structure of the $\beta$ phase of $\mathrm{ReO}_{2}$ [79].

\section{E. Nodal line arrangement in SGs 61 and 73}

Space group 61 is a supergroup of SG 19 with inversion as an additional generator. This adds further restrictions to the compatibility relations for bands along the rotation axes discussed in Sec. IV B.

In the spinless case, SG 61 also has a nodal plane trio enforced by the three screw rotations. Within the other mirror planes $k_{i}=0, i=x, y$ or $z$, there is an hourglass nodal line in between the twofold degeneracies on the intersections with the nodal planes. For example, in the plane $k_{x}=0$ bands form an hourglass dispersion for any path connecting the lines $\mathrm{Z}-\mathrm{T}$ and $\mathrm{T}-\mathrm{Y}$, where $\mathrm{T}$ is fourfold degenerate. This includes the path from $Z$ to $Y$ via $\Gamma$ along the rotation axes. The segments of this path are always part of two mirror planes and the dispersion along these axes has to accommodate for the eigenvalue relations of both symmetries simultaneously. No two bands paired at one of the TRIMs X, Y, or Z can be paired again at some other point. This enforces three crossings on two of the three lines. A possible arrangement is shown in Fig. 9(b). Each irreducible representation along the axis is completely determined by the two mirror eigenvalues of the planes meeting along this line. This is shown by the double lines, where the color indicates the positive (blue) or negative (orange) eigenvalue. Note that the order of colors is important. 
(a)

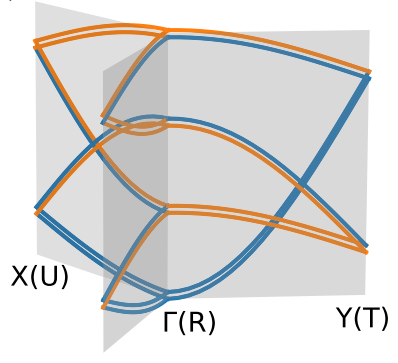

$\mathrm{Z}(\mathrm{S})$ (b)

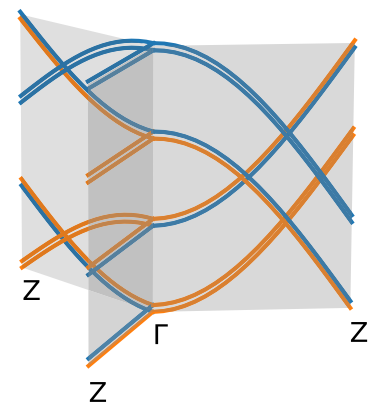

FIG. 9. Possible arrangement of bands along three rotation axes in (a) SG 61 in the spinless case for the TRIMs without brackets and the spinful case for the TRIMs in brackets, (b) SG 73 in the spinless case. Blue and orange mark the sign of both mirror eigenvalues on each axis according to Eq. (5.1).

A crossing can only be removed, when both eigenvalues are identical. Each crossing is part of an hourglass nodal line, extending into planes where the mirror eigenvalues differ. Exchanging the order of bands at $\Gamma$ moves the crossings to other symmetry axis, but cannot change the overall picture. The rotation eigenvalue is given by the product of both mirror eigenvalues. At $\Gamma$, the product of all rotation eigenvalues equals +1 as in SG 19, cf. Fig. 4(a).

In the spinful case, a similar situation unfolds on the BZ edges. There are three hourglass nodal lines, $(\mathrm{U}-\mathrm{X} ; \mathrm{S})$ in the $k_{x}=\pi$ plane, $(\mathrm{S}-\mathrm{Y} ; \mathrm{T})$ in the $k_{y}=\pi$ plane and $(\mathrm{T}-\mathrm{Z} ; \mathrm{U})$ in the $k_{z}=\pi$ plane. When connecting two TRIMs out of U,T and $S$ via $R$, the eigenvalues of the mirror symmetry whose invariant plane contains the three points need to exchange for every possible combination. For example, the path from $\mathrm{S}$ via $\mathrm{R}$ to $\mathrm{T}$ has to provide the eigenvalue exchange in the $k_{y}=\pi$ plane, but the segments $\mathrm{S}-\mathrm{R}$ and R-T must also be compatible with the hourglass nodal lines in the $k_{x}$ and $k_{z}$ planes, respectively. This is only possible with the same connectivity diagram for the three axis R-S, R-U, and R$\mathrm{T}$ as described above for the spinless case and shown in Fig. 4(a).

In SG 73, we also find an extension of the compatibility relations of its subgroup SG 24. All mirror symmetries are nonsymmorphic and their eigenvalues depend on $\mathbf{k}$. Consequently, an eigenvalue + changes into - when moving along a path which changes the corresponding coordinate by $2 \pi$ modulo reciprocal lattice translations. For example, the $M_{010}\left(0, \frac{1}{2}, \frac{1}{2}\right)$ eigenvalue +1 at $Z=(0,0,2 \pi)$ is labeled with + according to the definition in Eq. (5.1), whereas at the equivalent point $Z^{\prime}=(2 \pi, 0,0)$ it is the one labeled -, i.e., the bands with + and - have to exchange for any path connecting $\mathrm{Z}$ to itself with a net displacement in $k_{y}$ of $2 \pi$. At the same time, the TRIMs S, R, and T are the origin of almost movable nodal lines in the $k_{x}=0, k_{y}=0$, and $k_{z}=0$ planes, respectively, which facilitate this exchange. Because $\Gamma$ connects to $Z$ via all three rotation axes and the above considerations are valid for all three glide mirrors, there are fewer choices for arranging the states to avoid crossings. This leads to at least six crossings, all being part of nodal lines again. A possible arrangement is shown in Fig. 9(b).

\section{F. Almost movable Dirac line}

There is only one instance of an almost movable Dirac line in centrosymmetric orthorhombic SGs with SOC, namely in SG 63. Because $M_{001}\left(0,0, \frac{1}{2}\right)$ is off-centered, the representations $U_{M_{001}}$ and $U_{\mathcal{P}}$ anticommute in the $k_{z}=\pi$ plane and the mirror eigenvalues $\pm \mathrm{i}$ are paired as $(+,+)$ or $(-,-)$ by $\mathcal{P} \mathcal{T}$. At the same time, $\mathcal{T}$ relates $(+,+)$ at $\mathrm{R}+\left(q_{x}, q_{y}, 0\right)$ to $(-,-)$ at $\mathrm{R}-\left(q_{x}, q_{y}, 0\right)$, requiring the eigenvalues to exchange on any path in between these two points. This requires a fourfold degeneracy along a line through R. Following our notation in Sec. VE, we call this line an almost movable Dirac line. The same argument applies, in principle, to every other TRIM in the $k_{z}=\pi$ plane as well, but for them pinned nodal lines facilitate the exchange.

\section{Material examples}

The compound LiBH may prove to be a valuable realization of a material, where a twofold nodal line and a nodal plane with a pinned fourfold nodal line coexist in the vicinity of the Fermi energy [80]. Other known orthorhombic realizations of movable enforced nodal lines in the vicinity of the Fermi energy include $\mathrm{AgF}_{2}$ (SG 61) [81], $\mathrm{Ba}_{2} \mathrm{ReO}_{5}$ (SG 62) [73], and $\mathrm{SrIrO}_{3}$ (SG 62) [82]. Accidental nodal lines have been found for $\mathrm{BaLi}_{2} \mathrm{Sn}$ (SG 59) [83], $\mathrm{BaLi}_{2} \mathrm{Si}$ (SG 59) [84], $\mathrm{ZrAs}_{2}$ (SG 62) [85], $\mathrm{Ta}_{3} \mathrm{SiTe}_{6}$ and related compounds (SG 62) [86,87], as well as 3D $\alpha^{\prime}$ boron (SG 63) [88].

\section{G. Symmetry-enforced $\mathbb{Z}_{2}$ topology in planes}

Topologically nontrivial insulators can be understood by an inversion of bands, which means that the valence bands by themselves cannot be expressed as a sum of trivial elementary band representations [89]. Such a nontrivial topology is not limited to topological insulators, but can also be found, and even enforced, for gapped subsystems within a single set of connected bands [19,90,91].

In the following, we will discuss the centrosymmetric SGs 56,61 , and 62 with strong SOC that enforce a nontrivial weak topological invariant already within a minimal set of connected bands. To identify whether an effective band inversion must occur in a subset of the BZ, we calculate the $\mathbb{Z}_{2}$ index from inversion eigenvalues [92], which can be obtained for some SGs directly from the crystalline symmetries. Furthermore, the argument can be extended by including SGs for which a system inherits an actual band inversion from the case without spin-orbit coupling that persists with sufficiently small SOC.

First, we introduce the $\mathbb{Z}_{2}$ invariant and apply the formalism to SG 62, the symmetry group of $\operatorname{Ir}_{2} \mathrm{Si}$, for which a nontrivial weak invariant is enforced. We then discuss SG 52 for which a nontrivial topology follows from the case of vanishing $\mathrm{SOC}$, which is confirmed explicitly for $\mathrm{Sr}_{2} \mathrm{Bi}_{3}$.

The weak $\mathbb{Z}_{2}$ invariant can be calculated on a subset of the BZ that includes four TRIMs $\Gamma_{i}$. The invariant $v$ is given by $(-1)^{v}=\prod_{\Gamma_{i}} \delta_{\Gamma_{i}}$ with $\delta_{\Gamma_{i}}=\prod_{m=1}^{N} \xi_{2 m}\left(\Gamma_{i}\right)$, where the inversion eigenvalue $\xi_{2 m}\left(\Gamma_{i}\right)$ is taken for the band $2 m$ at the TRIM $\Gamma_{i} \in\{\Gamma, \mathrm{X}, \mathrm{Y}, \mathrm{Z}, \mathrm{U}, \mathrm{S}, \mathrm{T}, \mathrm{R}\}$ [92]. $N$ denotes the number of occupied bands. Taking only the even-numbered occupied bands is well-defined because time-reversal symmetry pairs 

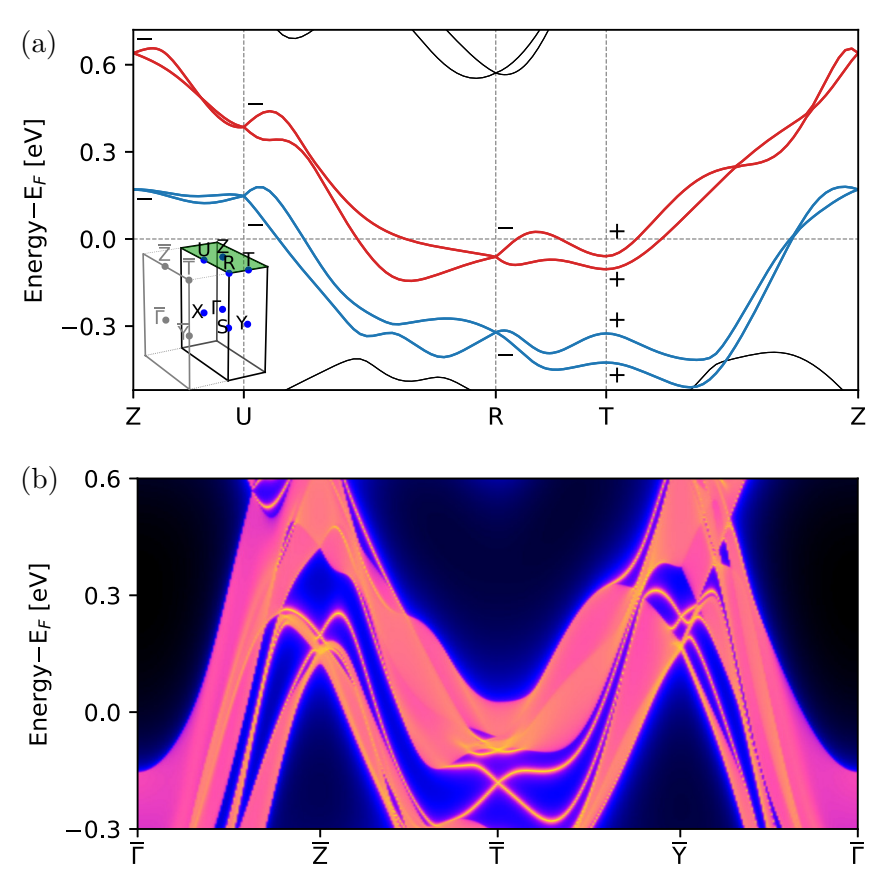

FIG. 10. (a) Bulk bands of $\mathrm{Sr}_{2} \mathrm{Bi}_{3}$ in the $k_{z}=\pi$ plane with the sign of the product of inversion eigenvalues for each set of band at all TRIMs in the plane. (b) Surface density of states on the (100) surface along a high-symmetry path in the surface BZ. The nontrivial plane maps on the line Z-T, see inset in (a), with symmetry enforced surface states crossing the projected bulk gap.

identical inversion eigenvalues $\xi_{2 m}\left(\Gamma_{i}\right)=\xi_{2 m+1}\left(\Gamma_{i}\right)$ at each TRIM $\Gamma_{i}$.

To illustrate the calculation of the inversion eigenvalues $\xi_{2 m}\left(\Gamma_{i}\right)$ and in extension the invariant $\nu$, we consider SG 62 as an example. In SG 62, all TRIMs except $\Gamma$ host fourfold degenerate Dirac points and thus we must consider $N=$ $2+4 \mathbb{N}_{0}$ occupied bands, for which $\delta_{\Gamma_{i}}$ can be well-defined. Note that for $N$ the bands are counted without their spin degeneracy, while we consider all the states in our discussion of inversion eigenvalues. A Dirac point may consist of either four identical inversion eigenvalues or two of each possible eigenvalue. If one takes into account that the mirror symmetry $M_{010}\left(0, \frac{1}{2}, 0\right)$ anticommutes with the inversion operation at TRIMs in the $k_{y}=\pi$ plane, one finds that these TRIMs contain both inversion eigenvalues, yielding $\xi_{2}(\{\mathrm{Y}, \mathrm{T}, \mathrm{R}, \mathrm{S}\})=$ $\pm 1=-\xi_{4}(\{\mathrm{Y}, \mathrm{T}, \mathrm{R}, \mathrm{S}\})$. An analogous argument holds for the mirror symmetry $M_{001}\left(\frac{1}{2}, 0, \frac{1}{2}\right)$, such that also at the TRIMs $\mathrm{Z}$ and $\mathrm{X}$ different eigenvalues are paired. Since $\Gamma$ does not host a Dirac point, only the TRIM U remains to be considered. At $\mathrm{U}$, the inversion commutes with the mirror symmetry $M_{010}\left(0, \frac{1}{2}, 0\right)$, and thus the bands can be labeled simultaneously by the eigenvalues of both symmetries. While the mirror symmetry $M_{001}\left(\frac{1}{2}, 0, \frac{1}{2}\right)$ commutes with inversion at $\mathrm{U}$, it anticommutes with $M_{010}\left(0, \frac{1}{2}, 0\right)$. The composite symmetry $\mathcal{T} M_{001}\left(\frac{1}{2}, 0, \frac{1}{2}\right)$ squares to -1 at $\mathrm{U}$ and relates the mirror eigenvalues as $(+,+)$ and $(-,-)$. Since time-reversal symmetry by itself pairs $(+,-)$, there are four distinct states. All four states carry the same inversion eigenvalue, because it is invariant under both $M_{001}\left(\frac{1}{2}, 0, \frac{1}{2}\right)$ and time reversal. These four orthogonal states form the Dirac point at U. In summary, for SG 62, we have found the inversion eigenvalues for all Dirac points at TRIMs with general arguments.

We can thus calculate the weak topological invariant for SG 62 using that $\delta_{\mathrm{X}}=\delta_{\mathrm{Y}}=\delta_{\mathrm{Z}}=\delta_{\mathrm{T}}=\delta_{\mathrm{S}}=\delta_{\mathrm{R}}=\xi_{2}(X) \xi_{4}(X)=$ $\pm 1(\mp 1)=-1$ and $\delta_{\mathrm{U}}=\xi_{2}(U) \xi_{4}(U)=+1$, which is true for both possible eigenvalues $\xi_{2}(U)=\xi_{4}(U)= \pm 1$. One concludes that the weak invariant of systems in SG 62 is fixed by symmetry, whereas the strong topology remains material dependent, because the order of bands at $\Gamma$ is not fixed. The latter would become relevant if there is a symmetry breaking perturbation that gaps the enforced nodal line (R-U-X;S) such that the system becomes insulating at the filling $N=2+4 \mathbb{N}_{0}$. Thus the weak topological invariant on any plane that contains $\mathrm{U}$, but neither $\Gamma$ nor the nodal line, is always nontrivial. This argument is independent of the specific order of bands and thus any material will exhibit the corresponding surface states in the gap that is also crossed by the nodal line (R-U-X;S). Let us consider for example the plane ZURT, where the invariant is given by $(-1)^{v}=\delta_{\mathrm{Y}} \delta_{\mathrm{T}} \delta_{\mathrm{R}} \delta_{\mathrm{S}}=-1$ and thus $v=1$ indicates a nontrivial subsystem. Due to the nonzero topological invariant $v$, there are surface states for any termination that truncates the plane ZURT, given that the projection of the bulk bands into the surface BZ does not close the gap. For example, for the (100) surface, there has to be a surface state that crosses a bulk gap between $\bar{Z}$ and $\overline{\mathrm{T}}$ in the surface BZ, while the bulk bands of the nodal line only close the gap in the vicinity of $\overline{\mathrm{Y}}$. We discuss $\mathrm{Ir}_{2} \mathrm{Si}$ as a material realization for SG 62 in Sec. VI G 2.

The described process for SG 62 also applies analogously to SGs 56 and 61, where SG 61 is a special case because there is no Dirac point at R. Nevertheless, the weak invariant is independent of the band order, because without SOC at R there is only one Dirac point per minimal set of connected bands, for which all inversion eigenvalues are identical. With SOC this degeneracy of identical inversion eigenvalues splits into four bands with the same representation regarding inversion. We have listed the nontrivial planes in terms of their TRIMs in the column "Nontrivial planes", the TRIM with identical eigenvalue pairing is highlighted in bold font.

In the following, we extend the previous approach to consider cases, where the order of inversion eigenvalues is fixed without SOC, which leads to a nontrivial weak invariant with SOC as long as SOC is sufficiently small, such that the bands are not inverted. We shall consider SG 52, for which by similar arguments as before the inversion eigenvalues for the occupied bands must lead to $\delta_{\mathrm{X}}=\delta_{\mathrm{Y}}=\delta_{\mathrm{Z}}=\delta_{\mathrm{U}}=\delta_{\mathrm{S}}=\delta_{\mathrm{R}}=$ $\xi_{2}(X) \xi_{4}(X)= \pm 1(\mp 1)=-1$ and $\delta_{\mathrm{T}}=1$ without SOC. Once SOC is added to the system, the Dirac point at $\mathrm{T}$ splits into two bands, but as long as no band inversion occurs the value of $\delta_{\mathrm{T}}$ does not change. Note that this induced arrangement of inversion eigenvalues is less stable than for SG 61, where a set of connected bands inherits only identical inversion eigenvalues from the case without SOC. In other words, for SG 52 an exchange of energy levels within a set of connected bands may trivialize the $\mathbb{Z}_{2}$ invariant, whereas for SG 61 such an exchange would not affect the topology. The topological invariant $v$ induced by SG 52 is $v=1$ for any plane that contains $T$ but neither $\Gamma$ nor the movable Dirac point on X-S. An analogous argument also holds for SG 60. 

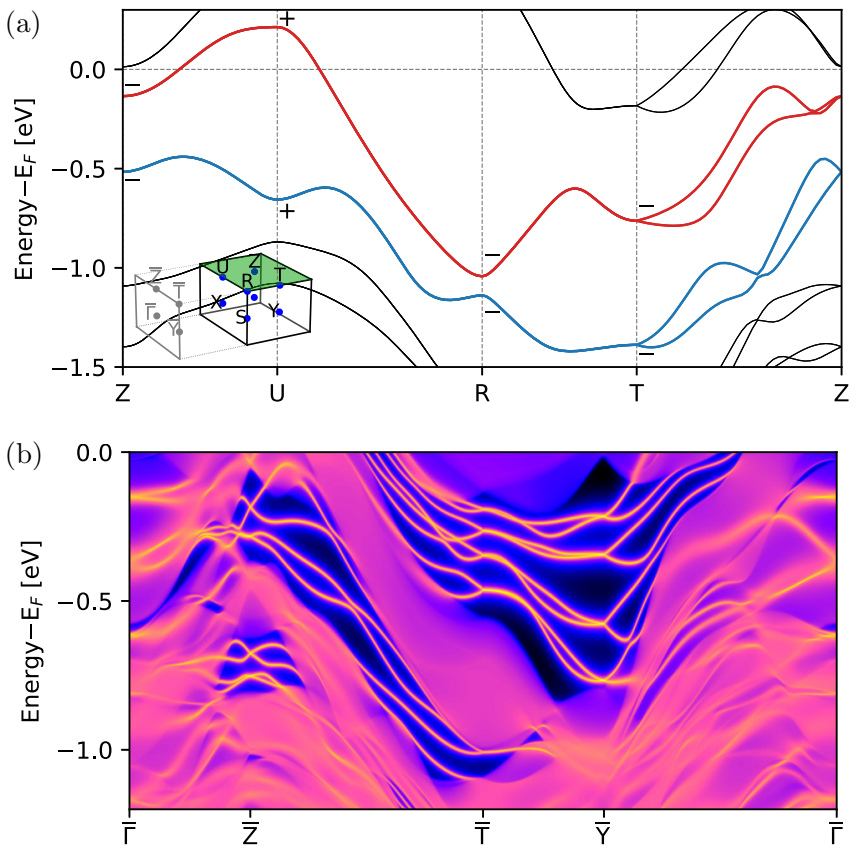

FIG. 11. (a) Bulk bands of $\operatorname{Ir}_{2} \mathrm{Si}$ in the $k_{z}=\pi$ plane with the sign of the product of of inversion eigenvalues for each set of band at all TRIMs in the plane. (b) Surface density of states on the (100)surface, where the nontrivial plane maps on the line Z-T, see inset in (a). A pair of time-reversal symmetric surface states connect the bands below the projected bulk gap to the upper ones.

To show the $\mathbb{Z}_{2}$ topology inherited from the case without SOC more clearly, we discuss the material $\mathrm{Sr}_{2} \mathrm{Bi}_{3}$ corresponding to SG 52 in Sec. VI G 1. In Sec. VI G 2, we present a material in SG 62, namely, $\mathrm{Ir}_{2} \mathrm{Si}$, which has a weak $\mathbb{Z}_{2}$ invariant enforced by symmetry alone.

\section{Material example: $\mathrm{Sr}_{2} \mathrm{Bi}_{3}$}

$\mathrm{Sr}_{2} \mathrm{Bi}_{3}$ crystallizes in SG 52 and was prepared by melting the elements with a long, subsequent annealing step [93]. It is very prone to oxidation in air, as expected based on the $\mathrm{Bi}-\mathrm{Bi}$ bonding. Growth of large single crystals may be challenging as it appears to be incongruently melting with a small exposed liquidus [94].

Projecting the bulk bands in [100] direction preserves the gap between conduction and valence bands in the ZURT plane, cf. the band structure along the high-symmetry path in Fig. 12(e). The nontrivial topology in that plane enforces surface states crossing that gap in the projection of the plane, see Fig. 10. Because time-reversal symmetry is preserved, the surface states form a Dirac point at TRIMs.

\section{Material example: $\mathrm{Ir}_{2} \mathrm{Si}$}

Although some ternary iridium silicides have attracted attention for superconductivity or heavy fermion behavior, very little is known about the binary $\operatorname{Ir}_{2}$ Si crystallizing in SG 62 . The initially reported structure [95] has been corroborated at least once [96]. In the first four bands below the Fermi energy, there is a gap between pairs of bands in the $k_{z}=\pi$ plane with symmetry enforced nontrivial $\mathbb{Z}_{2}$ invariant, shown in Fig. 11(a) and in the band structure along the full high- symmetry path in Fig. 12(f). The bulk gap of this plane is preserved for a (100) termination and the nontrivial topology enforces a surface state traversing the gap on the line $\bar{Z}-\bar{T}$, see Fig. 11(b). There are several additional surface states forming Dirac cones in the bulk gaps. Nevertheless, a clear signature of the nontrivial topology is the surface band that detaches from the lower bands at the center of the line $\overline{\mathrm{Z}}-\overline{\mathrm{T}}$, crosses the gap and connects to higher bands. In the process the partners of Kramers pairs at TRIMs change when comparing $\bar{Z}$ and $\bar{T}$, which would not be the case for trivial surface states.

\section{CONCLUSION}

In summary, we have classified all symmetry-enforced band topologies in orthorhombic crystals with time-reversal symmetry, both in the presence and absence of spinorbit coupling. We have found a vast number of different symmetry-enforced band crossings, as well as enforced weak $\mathbb{Z}_{2}$ invariants (Tables I-IV). Weyl points exist in the absence of inversion and are enforced to occur by Kramers theorem (or generalizations thereof), by off-centered rotations (Sec. IV A), or by screw rotations (Secs. IV B and V A). Fourfold double Weyl points with $|\mathcal{C}|=2$ exist in the presence of several screw rotations combined with time-reversal symmetry (Sec. IV D). Dirac points at high-symmetry points are enforced by the combination of inversion with time-reversal (Sec. VI A), while movable Dirac points arise in the presence of screw rotations together with space-time inversion $\mathcal{P} \mathcal{T}$ (Sec. VIB).

Line degeneracies can be enforced by orthorhombic symmetries as well. For example, movable Weyl lines with hourglass dispersion are generated by glide mirror symmetries (Sec. V C). These Weyl lines can form nodal chains if several glide mirror symmetries with orthogonal mirror planes are present (Sec. V D). We find that such nodal chains exist in the bands of CuIrB near the Fermi level. Almost movable lines occur in the presence of mirror symmetries whose mirror planes contain TRIMs without any additional symmetries (Sec. V E). Movable Dirac lines are enforced by mirror symmetries that are off-centered with respect to space-time inversion $\mathcal{P} \mathcal{T}$ (Sec. VID).

Finally, orthorhombic symmetries can also enforce nodal planes, i.e., two-dimensional degeneracies of the bands at the boundary of the BZ. These are enforced by the combination of screw rotations with time-reversal symmetry (Sec. IV C). Interestingly, in SG 19 with SOC there are three such nodal planes (i.e., a nodal plane trio) which are sources (or sinks) of quantized Berry flux, which is absorbed (emitted) by a single Weyl point at $\Gamma$.

Besides band degeneracies, orthorhombic symmetries can also enforce nontrivial $\mathbb{Z}_{2}$ topologies in two-dimensional, gapped subspaces of the three-dimensional BZ. The corresponding $\mathbb{Z}_{2}$ indices are computed from the inversion eigenvalues at the TRIMs within the two-dimensional subspaces, whose values are fixed by symmetry in SGs 56 and 62 , as well as in SG 61 provided that elementary band representations with different inversion eigenvalues do not mix. Similarly, we find that also SGs 52 and 60 enforce weak $\mathbb{Z}_{2}$ invariants, however, only in the case of weak SOC that does not invert the bands. We have identified two materials, namely $\mathrm{Ir}_{2} \mathrm{Si}$ (SG 62) and $\mathrm{Sr}_{2} \mathrm{Bi}_{3}$ (SG 52), with such 
(a) $\mathrm{Pd}_{7} \mathrm{Se}_{4}$

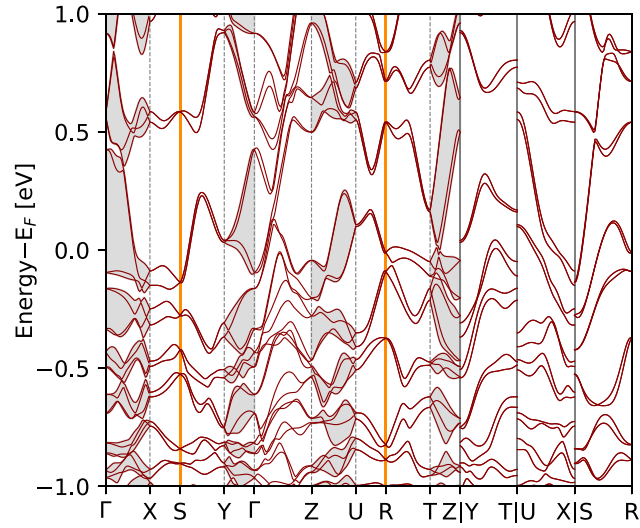

(c) AuTlSb

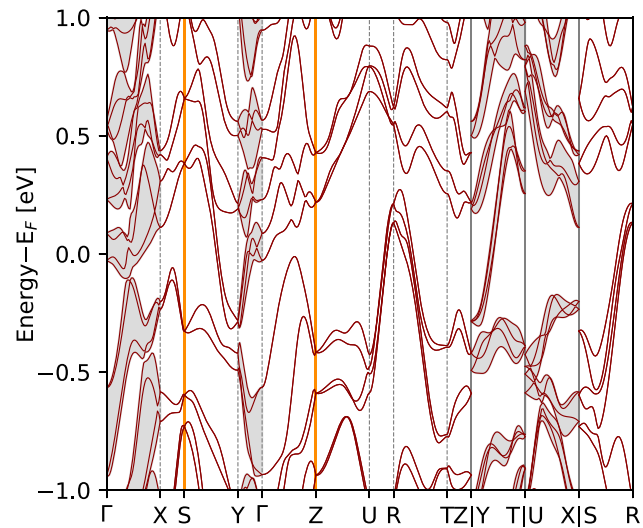

(e) $\mathrm{Sr}_{2} \mathrm{Bi}_{3}$

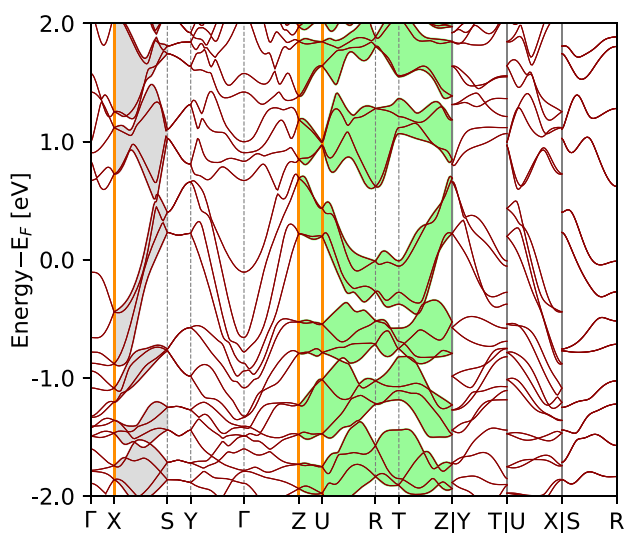

(b) $\mathrm{Ag}_{2} \mathrm{Se}$

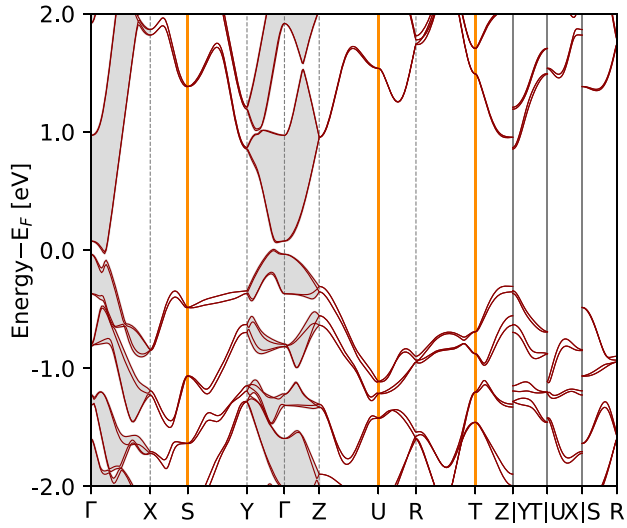

(d) $\mathrm{CuIrB}$

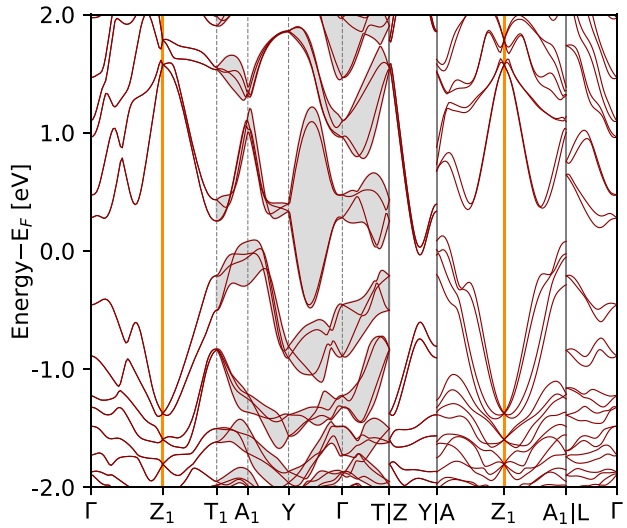

(f) $\operatorname{Ir}_{2} \mathrm{Si}$

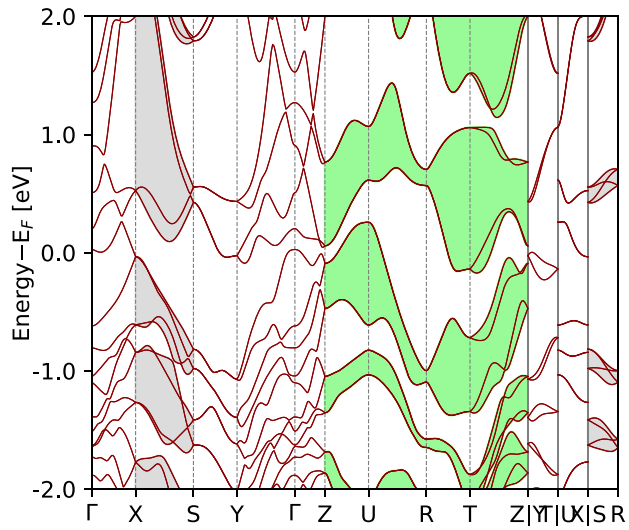

FIG. 12. DFT band structures including SOC along the high-symmetry paths for all examples discussed in the main text. The positions of symmetry-enforced fourfold degenerate points are highlighted in orange and correspond to fourfold double Weyl points, fourfold degenerate points with $\mathcal{C}=0$ and Dirac points in the first, second and third row, respectively. The movable fourfold double Weyl points in (b) are highlighted with arrows. Gray areas highlight connected bands in path segments with an hourglass dispersion. Green areas indicate the set of connected bands in a plane with nontrivial $\mathbb{Z}_{2}$-invariant.

symmetry-enforced weak invariants, which lead to surface helical modes of topological origin.

Interestingly, there are also some orthorhombic SGs, where the number of Weyl points of a given band pair can be as low as two. For example, in SGs 36, 45, and 46 with SOC, there can be only two Weyl points at two TRIMs, while the other TRIMs are part of nodal planes or nodal lines. Remarkably, in SG 24 without SOC a given band pair can have only four Weyl points, while the TRIMs are nondegenerate. In SG 19 without
SOC, there are only three Weyl points for an even number of filled bands, one of which is a fourfold double Weyl point.

Furthermore, we provide an example of how to use our purely symmetry-based analysis as a guide to identifying interesting new materials. The screening criteria mentioned in Sec. III can be used as a starting point for identifying candidates in known compounds, which in turn might act as a starting point for synthesis of related new compounds of the same SG. By explicitly including spinless band structures in 


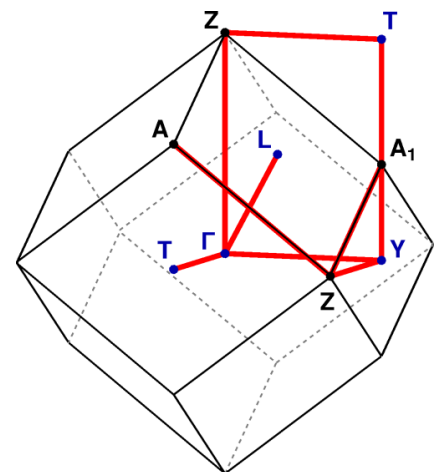

FIG. 13. High-symmetry path for CuIrB in SG 43 used in Fig. 12(d).

our analysis, our paper also applies to phonon bands and can serve as a blueprint for constructing synthetic materials, e.g., photonic crystals, acoustic metamaterials or electric circuit networks. The methods used have also been applied to the hexagonal, trigonal, and tetragonal SGs [17-19] and can be extended to further SGs and, in a similar manner, to magnetic SGs. Another topic for further investigations is the question of which symmetries, irreducible band representations, and band orderings lead to enforced higher-order topological insulators [90,97-99] or semimetals [100-103] with corner states.

\section{ACKNOWLEDGMENTS}

The authors thank K. Alpin and W. Yau for useful discussions. D.H.F. gratefully acknowledges financial support from the Alexander von Humboldt Foundation.

\section{APPENDIX A: ADDITIONAL BAND STRUCTURE CALCULATIONS}

For all the example materials, we present in this Appendix the band structure along the full high-symmetry path, aligned with the symmetries as specified in Sec. II. We use the standard path for all SGs with a primitive lattice, see Fig. 1(a). Note that the path for CuIrB extends beyond the first full BZ to show the full line $T_{1}-Y$ via $A_{1}$, where the nodal chain is formed. See Fig. 13 for the definition.

For each plot, we highlight the path segments with an hourglass dispersion by coloring the space between bands in gray, i.e., the hourglass dispersion appears always within the gray area.

For the two SGs with crystallographic point group 222, Figs. 12(a) and 12(b), the hourglass dispersion always enforces a movable Weyl point and the points highlighted by orange lines host fourfold double Weyl points in all bands, see Sec. IV D.

Figures 12(c) and 12(d) contain the two example materials with crystallographic point group $m m 2$. The hourglass dispersion there belongs always to movable Weyl lines and the fourfold degenerate points carry no Chern number, as described in Sec. V F.

The two materials shown at the bottom of Fig. 12 are centrosymmetric and all bands are twofold degenerate. The points highlighted in orange host pinned Dirac points. The hourglass dispersion belongs to a movable Dirac point in Fig. 12(e) and to a movable Dirac line in Fig. 12(f). Additionally, all bands belonging to a set of connected bands with a nontrivial $\mathbb{Z}_{2-}$ invariant are filled in with green in the corresponding plane. For these two examples, there is in principal another possible plane running diagonally through the BZ. However, since no path segment lies within this plane it is not shown.

\section{APPENDIX B: LOW-ENERGY HAMILTONIANS FOR FOURFOLD DEGENERATE POINTS}

Here we give low-energy Hamiltonians for the fourfold double Weyl points with Chern number \pm 2 , and the fourfold degenerate points formed by intersecting nodal lines, either with an additional hourglass nodal line in between or as true point defects.

\section{Fourfold double Weyl point}

To define the Hamiltonians describing fourfold double Weyl points, we use the Pauli matrices $\sigma_{i}$ and $\tau_{i}(i=0, x, y, z)$, acting in spin and orbital space, respectively.

The little group of a fourfold double Weyl point consists of the three twofold rotations of the chiral orthorhombic SGs and time-reversal symmetry. Their representations can be given as

$$
\begin{gathered}
U_{2_{001}}=\mathrm{i} \tau_{0} \tau_{z}, \\
U_{2_{010}}=\tau_{0} \tau_{y}, \\
U_{2_{100}}=\tau_{0} \tau_{x}, \\
U_{\mathcal{T}} \mathcal{K}=\mathrm{i} \tau_{y} \sigma_{x} \mathcal{K} .
\end{gathered}
$$

Since two rotations are screw rotations, their eigenvalues are \pm 1 , consistent with the requirement of squaring to the identity.

The Hamiltonian up to linear order in $\mathbf{q}$ around the TRIM $\mathbf{K}$, i.e., $\mathbf{q}=\mathbf{k}-\mathbf{K}$, invariant under the above symmetries is restricted to the form

$$
\begin{aligned}
H(\mathbf{q})= & v_{x} q_{x} \tau_{z} \sigma_{x}+v_{y} q_{y} \tau_{z} \sigma_{y}+v_{z} q_{z} \tau_{0} \sigma_{z} \\
& +\lambda_{x} q_{x} \tau_{x} \sigma_{x}+\lambda_{y} q_{y} \tau_{x} \sigma_{y} .
\end{aligned}
$$

The real parameters $v_{i}$ define the velocities or slope of the individual Weyl points. The splitting from SOC is introduced from the real parameters $\lambda_{i}$, which renormalize the velocities $v_{i}$. The Chern number of this Hamiltonian is \pm 2 and it reproduces the twofold degenerate nodal planes for $q_{x}=0$ or $q_{y}=0$

\section{Fourfold degenerate points from multiple intersecting nodal lines}

As discussed in Sec. VF, fourfold degeneracies in the rhombic pyramidal SGs are formed by intersecting nodal lines and can either be pointlike or have an hourglass nodal line running through the fourfold degeneracy. 
For the fourfold degenerate point, the representations for the generators at the TRIM can be given by

$$
\begin{gathered}
U_{2_{001}}=-\mathrm{i} \tau_{0} \sigma_{z}, \\
U_{M_{010}}=\tau_{0} \sigma_{y}, \\
U_{\mathcal{T}} \mathcal{K}=\mathrm{i} \tau_{y} \sigma_{x} \mathcal{K},
\end{gathered}
$$

and the linearized Hamiltonian is restricted to the form

$$
\begin{aligned}
H(\mathbf{q})= & v_{z} q_{z} \tau_{z} \sigma_{0}+v_{x} q_{x} \tau_{z} \sigma_{y}+v_{y} q_{y} \tau_{z} \sigma_{x} \\
& +\lambda_{x} q_{x} \tau_{x} \sigma_{y}+\lambda_{y} q_{y} \tau_{x} \sigma_{x}+\lambda_{z} q_{z} \tau_{x} \sigma_{0},
\end{aligned}
$$

with the real parameters $v_{i}$ and $\lambda_{i}$. The gap between the second and third band closes only at the TRIM, i.e., for $\mathbf{q}=0$, but the Chern number vanishes. In the case of an hourglass nodal line, the representations are

$$
\begin{gathered}
U_{2_{001}}=\tau_{0} \sigma_{z}, \\
U_{M_{010}}=\tau_{0} \sigma_{y}, \\
U_{\mathcal{T}} \mathcal{K}=\mathrm{i} \tau_{y} \sigma_{z} \mathcal{K},
\end{gathered}
$$

and the linearized Hamiltonian reads then

$$
\begin{aligned}
H(\mathbf{q})= & v_{z} q_{z} \tau_{z} \sigma_{0}+v_{x} q_{x} \tau_{z} \sigma_{y}+v_{y} q_{y} \tau_{0} \sigma_{x} \\
& +\lambda_{x} q_{x} \tau_{x} \sigma_{y}+\lambda_{z} q_{z} \tau_{x} \sigma_{0},
\end{aligned}
$$

again with purely real parameters $v_{i}$ and $\lambda_{i}$. There are twofold degeneracies on the axes $\left(q_{x}, 0,0\right)$ and $\left(0,0, q_{z}\right)$ and an additional hourglass nodal line in between the second and third band, running through $\mathbf{q}=0$.
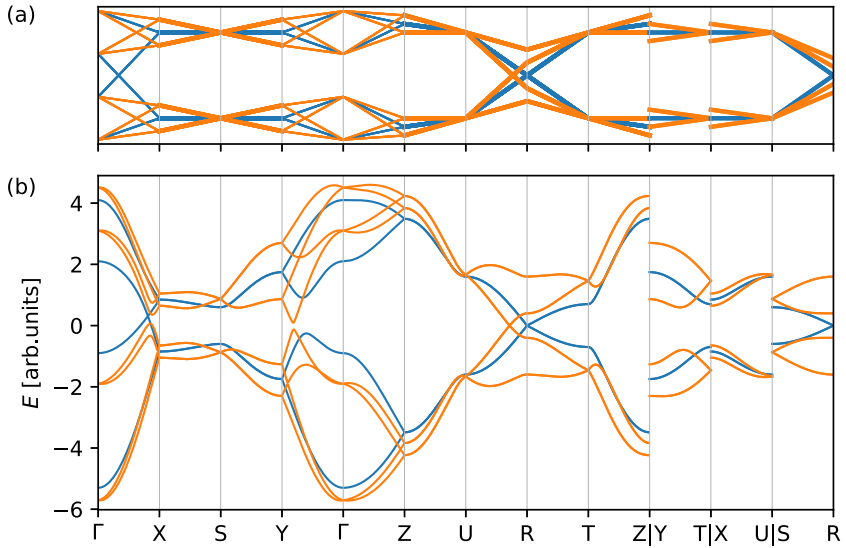

FIG. 14. Elementary bands representations in SG 19 without SOC (blue) and with strong SOC (orange). The upper bands are a schematic band structure with minimal number of crossings, based on compatibility relations alone. The lower set of bands are from the minimal tight-binding model defined in Eq. (C1) with hopping parameters $t_{0}=-t_{5}=0.25, t_{1}=-t_{4}=0.55, t_{2}=-0.6, t_{3}=$ -0.95 , and $l_{i}=0$ for vanishing SOC. For the case with SOC $l_{0}=$ $l_{1}=l_{7}=0, l_{2}=-l_{5}=0, l_{3}=0.7, l_{4}=-l_{8}=0.2, l_{5}=-0.3, l_{9}=$ $l_{10}=-l_{11}=0.1$. Parameters were chosen for maximal visibility of the features mentioned in the text.

\section{APPENDIX C: MINIMAL TIGHT-BINDING MODEL FOR SG 19}

SG 19 enforces all notable features of chiral SGs, namely Kramers and movable Weyl points, fourfold double Weyl points and topological nodal planes. Therefore we present a minimal tight-binding model to give a simple example and to demonstrate the connection between spinless and spinful band structures.

The minimal number of sites is four per unit cell and we include nearest neighbor hopping only. This implicitly excludes terms in the Hamiltonian proportional to $\mathbb{1}$, i.e., a uniform dispersion for the bands and lets us focus on the splitting of bands.

$$
\begin{aligned}
& H(\mathbf{k})=\left(\begin{array}{cc}
H_{0}(\mathbf{k}) & \Lambda(\mathbf{k}) \\
\Lambda^{\dagger}(\mathbf{k}) & H_{0}^{*}(-\mathbf{k})
\end{array}\right),
\end{aligned}
$$

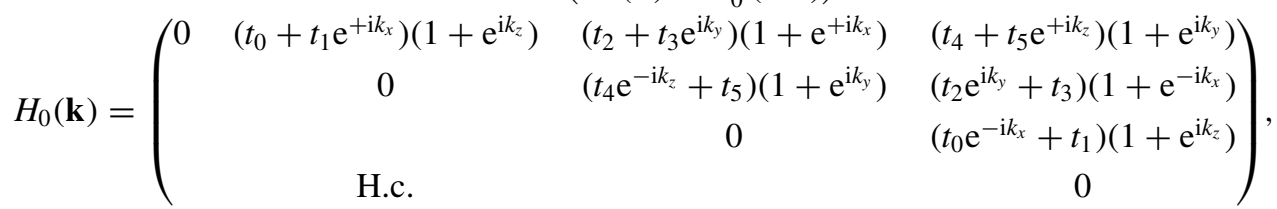

$$
\begin{aligned}
& \Lambda(\mathbf{k})=\left(\begin{array}{cccc}
(0 & \left(l_{0}+l_{1} \mathrm{e}^{+\mathrm{i} k_{x}}\right)\left(1+\mathrm{e}^{+\mathrm{i} k_{z}}\right) & \left(l_{2}+l_{3} \mathrm{e}^{+\mathrm{i} k_{y}}\right)\left(1+\mathrm{e}^{+\mathrm{i} k_{x}}\right) & \left(l_{4}+l_{5} \mathrm{e}^{+\mathrm{i} k_{z}}\right)\left(1-\mathrm{e}^{+\mathrm{i} k_{y}}\right) \\
-\left(l_{0}+l_{1} \mathrm{e}^{-\mathrm{i} k_{x}}\right)\left(1+\mathrm{e}^{-\mathrm{i} k_{z}}\right) & 0 & \left(l_{4} \mathrm{e}^{-\mathrm{i} k_{z}}+l_{5}\right)\left(1-\mathrm{e}^{+\mathrm{i} k_{y}}\right) & -\left(l_{2} \mathrm{e}^{+\mathrm{i} k_{y}}+l_{3}\right)\left(1+\mathrm{e}^{-\mathrm{i} k_{x}}\right) \\
-\left(l_{2}+l_{3} \mathrm{e}^{-\mathrm{i} k_{y}}\right)\left(1+\mathrm{e}^{-\mathrm{i} k_{x}}\right) & -\left(l_{4} \mathrm{e}^{+\mathrm{i} k_{z}}+l_{5}\right)\left(1-\mathrm{e}^{-\mathrm{i} k_{y}}\right) & 0 & -\left(l_{0} \mathrm{e}^{-\mathrm{i} k_{x}}+l_{1}\right)\left(1+\mathrm{e}^{+\mathrm{i} k_{z}}\right) \\
-\left(l_{4}+l_{5} \mathrm{e}^{-\mathrm{i} k_{z}}\right)\left(1-\mathrm{e}^{-\mathrm{i} k_{y}}\right) & \left(l_{2} \mathrm{e}^{-\mathrm{i} k_{y}}+l_{3}\right)\left(1+\mathrm{e}^{+\mathrm{i} k_{x}}\right) & \left(l_{0} \mathrm{e}^{+\mathrm{i} k_{x}}+l_{1}\right)\left(1+\mathrm{e}^{-\mathrm{i} k_{z}}\right) & 0
\end{array}\right) \\
& +\mathrm{i}\left(\begin{array}{cccc}
0 & \left(l_{6}+l_{7} \mathrm{e}^{+\mathrm{i} k_{x}}\right)\left(1+\mathrm{e}^{+\mathrm{i} k_{z}}\right) & \left(l_{8}+l_{9} \mathrm{e}^{+\mathrm{i} k_{y}}\right)\left(1-\mathrm{e}^{+\mathrm{i} k_{x}}\right) & -\left(l_{10}+l_{11} \mathrm{e}^{+\mathrm{i} k_{z}}\right)\left(1+\mathrm{e}^{+\mathrm{i} k_{y}}\right) \\
-\left(l_{6}+l_{7} \mathrm{e}^{-\mathrm{i} k_{x}}\right)\left(1+\mathrm{e}^{-\mathrm{i} k_{z}}\right) & 0 & \left(l_{10} \mathrm{e}^{-\mathrm{i} k_{z}}+l_{11}\right)\left(1+\mathrm{e}^{+\mathrm{i} k_{y}}\right) & -\left(l_{8} \mathrm{e}^{+\mathrm{i} k_{y}}+l_{9}\right)\left(1-\mathrm{e}^{-\mathrm{i} k_{x}}\right) \\
-\left(l_{8}+l_{9} \mathrm{e}^{-\mathrm{i} k_{y}}\right)\left(1-\mathrm{e}^{-\mathrm{i} k_{x}}\right) & -\left(l_{10} \mathrm{e}^{+\mathrm{i} k_{z}}+l_{11}\right)\left(1+\mathrm{e}^{-\mathrm{i} k_{y}}\right) & 0 & \left(l_{6} \mathrm{e}^{-\mathrm{i} k_{x}}+l_{7}\right)\left(1+\mathrm{e}^{+\mathrm{i} k_{z}}\right) \\
\left(l_{10}+l_{11} \mathrm{e}^{-\mathrm{i} k_{z}}\right)\left(1+\mathrm{e}^{-\mathrm{i} k_{y}}\right) & \left(l_{8} \mathrm{e}^{-\mathrm{i} k_{y}}+l_{3}\right)\left(1-\mathrm{e}^{+\mathrm{i} k_{x}}\right) & -\left(l_{6} \mathrm{e}^{+\mathrm{i} k_{x}}+l_{7}\right)\left(1+\mathrm{e}^{-\mathrm{i} k_{z}}\right) & 0
\end{array}\right) .
\end{aligned}
$$


Within each spin subspace, there are six independent real hopping parameters $t_{i}$ in $H_{0}(\mathbf{k})$ and its time-reversal symmetric copy $H_{0}^{*}(-\mathbf{k})$. Including SOC adds additional 12 real parameters $l_{i}$ in the off-diagonal block $\Lambda(\mathbf{k})$.

In Fig. 14, we present a schematic and exemplary band structure along the standard high-symmetry path, which can be compared to the material example $\mathrm{Ag}_{2} \mathrm{Se}$ in the same $\mathrm{SG}$ shown in Fig. 12(b). The nodal planes can be seen in the twofold degenerate bands on all paths except for the ones in the interior of the BZ, i.e., the ones connecting to $\Gamma$. On one of these paths, a band crossing needs to exist in the absence of
SOC. In the example, it can be observed on $\Gamma-X$. The TRIM $\mathrm{R}$ is fourfold degenerate.

Switching on SOC lifts spin degeneracy almost everywhere. The nodal planes and TRIMs remain at least twofold degenerate, while the degeneracy at $\mathrm{R}$ is now only twofold, the TRIMs S, U, and T and a movable point on the U-R axis remain fourfold degenerate. Further Weyl points are introduced in the hourglass dispersion along the rotation axes $\Gamma-\mathrm{X}, \Gamma-\mathrm{Y}$, and $\Gamma-Z$. Because of unbalanced Weyl points at $\Gamma$, the nodal planes are topological, i.e., act as source or sink of Berry flux.
[1] D. Hsieh, D. Qian, L. Wray, Y. Xia, Y. S. Hor, R. J. Cava, and M. Z. Hasan, A topological Dirac insulator in a quantum spin hall phase, Nature (London) 452, 970 (2008).

[2] D. Hsieh, Y. Xia, L. Wray, D. Qian, A. Pal, J. H. Dil, J. Osterwalder, F. Meier, G. Bihlmayer, C. L. Kane, Y. S. Hor, R. J. Cava, and M. Z. Hasan, Observation of unconventional quantum spin textures in topological insulators, Science 323, 919 (2009).

[3] M. Z. Hasan and C. L. Kane, Colloquium: Topological insulators, Rev. Mod. Phys. 82, 3045 (2010).

[4] A. P. Schnyder, S. Ryu, A. Furusaki, and A. W. W. Ludwig, Classification of topological insulators and superconductors in three spatial dimensions, Phys. Rev. B 78, 195125 (2008).

[5] A. Kitaev, Periodic table for topological insulators and superconductors, AIP Conf. Proc. 1134, 22 (2009).

[6] C.-K. Chiu, J. C. Y. Teo, A. P. Schnyder, and S. Ryu, Classification of topological quantum matter with symmetries, Rev. Mod. Phys. 88, 035005 (2016).

[7] Y. Ando and L. Fu, Topological crystalline insulators and topological superconductors: From concepts to materials, Annu. Rev. Condens. Matter Phys. 6, 361 (2015).

[8] C.-K. Chiu, H. Yao, and S. Ryu, Classification of topological insulators and superconductors in the presence of reflection symmetry, Phys. Rev. B 88, 075142 (2013).

[9] C.-K. Chiu and A. P. Schnyder, Classification of reflectionsymmetry-protected topological semimetals and nodal superconductors, Phys. Rev. B 90, 205136 (2014).

[10] B. Yan and C. Felser, Topological materials: Weyl semimetals, Annu. Rev. Condens. Matter Phys. 8, 337 (2017).

[11] T. Morimoto and A. Furusaki, Topological classification with additional symmetries from Clifford algebras, Phys. Rev. B 88, 125129 (2013).

[12] Y. X. Zhao, A. P. Schnyder, and Z. D. Wang, Unified Theory of $P T$ and $C P$ Invariant Topological Metals and Nodal Superconductors, Phys. Rev. Lett. 116, 156402 (2016).

[13] X.-Y. Song and A. P. Schnyder, Interaction effects on the classification of crystalline topological insulators and superconductors, Phys. Rev. B 95, 195108 (2017).

[14] M. Stone, C.-K. Chiu, and A. Roy, Symmetries, dimensions and topological insulators: the mechanism behind the face of the Bott clock, J. Phys. A: Math. Theor. 44, 045001 (2010).

[15] J. Kruthoff, J. de Boer, J. van Wezel, C. L. Kane, and R.-J. Slager, Topological Classification of Crystalline Insulators Through Band Structure Combinatorics, Phys. Rev. X 7 , 041069 (2017).
[16] L. Elcoro, B. Bradlyn, Z. Wang, M. G. Vergniory, J. Cano, C. Felser, B. A. Bernevig, D. Orobengoa, G. de la Flor, and M. I. Aroyo, Double crystallographic groups and their representations on the Bilbao Crystallographic Server, J. Appl. Crystallogr. 50, 1457 (2017).

[17] J. Zhang, Y.-H. Chan, C.-K. Chiu, M. G. Vergniory, L. M. Schoop, and A. P. Schnyder, Topological band crossings in hexagonal materials, Phys. Rev. Materials 2, 074201 (2018).

[18] Y.-H. Chan, B. Kilic, M. M. Hirschmann, C.-K. Chiu, L. M. Schoop, D. G. Joshi, and A. P. Schnyder, Symmetry-enforced band crossings in trigonal materials: Accordion states and Weyl nodal lines, Phys. Rev. Materials 3, 124204 (2019).

[19] M. M. Hirschmann, A. Leonhardt, B. Kilic, D. H. Fabini, and A. P. Schnyder, Symmetry-enforced band crossings in tetragonal materials: Dirac and Weyl degeneracies on points, lines, and planes, Phys. Rev. Materials 5, 054202 (2021).

[20] T. Bzdušek, Q. Wu, A. Rüegg, M. Sigrist, and A. A. Soluyanov, Nodal-chain metals, Nature (London) 538, 75 (2016).

[21] A. Furusaki, Weyl points and Dirac lines protected by multiple screw rotations, Science Bulletin 62, 788 (2017).

[22] R. Takahashi, M. Hirayama, and S. Murakami, Spinless hourglass nodal-line semimetals, Phys. Rev. B 96, 155206 (2017).

[23] B.-J. Yang, T. A. Bojesen, T. Morimoto, and A. Furusaki, Topological semimetals protected by off-centered symmetries in nonsymmorphic crystals, Phys. Rev. B 95, 075135 (2017).

[24] C. Fang, M. J. Gilbert, X. Dai, and B. A. Bernevig, Multi-Weyl Topological Semimetals Stabilized by Point Group Symmetry, Phys. Rev. Lett. 108, 266802 (2012).

[25] S. S. Tsirkin, I. Souza, and D. Vanderbilt, Composite Weyl nodes stabilized by screw symmetry with and without timereversal invariance, Phys. Rev. B 96, 045102 (2017).

[26] F. Tang, H. C. Po, A. Vishwanath, and X. Wan, Topological materials discovery by large-order symmetry indicators, Sci. Adv. 5, eaau8725 (2019).

[27] B. Bradlyn, L. Elcoro, J. Cano, M. G. Vergniory, Z. Wang, C. Felser, M. I. Aroyo, and B. A. Bernevig, Topological quantum chemistry, Nature (London) 547, 298 (2017).

[28] M. G. Vergniory, L. Elcoro, Z. Wang, J. Cano, C. Felser, M. I. Aroyo, B. A. Bernevig, and B. Bradlyn, Graph theory data for topological quantum chemistry, Phys. Rev. E 96, 023310 (2017).

[29] https://icsd.fiz-karlsruhe.de/.

[30] A. Jain, S. P. Ong, G. Hautier, W. Chen, W. D. Richards, S. Dacek, S. Cholia, D. Gunter, D. Skinner, G. Ceder, and K. a. 
Persson, The Materials Project: A materials genome approach to accelerating materials innovation, APL Mater. 1, 011002 (2013).

[31] S. P. Ong, S. Cholia, A. Jain, M. Brafman, D. Gunter, G. Ceder, and K. A. Persson, The materials application programming interface (API): A simple, flexible and efficient API for materials data based on REpresentational State Transfer (REST) principles, Comput. Mater. Sci. 97, 209 (2015).

[32] T. Zhang, Y. Jiang, Z. Song, H. Huang, Y. He, Z. Fang, H. Weng, and C. Fang, Catalogue of topological electronic materials, Nature (London) 566, 475 (2019).

[33] M. G. Vergniory, L. Elcoro, C. Felser, N. Regnault, B. A. Bernevig, and Z. Wang, A complete catalogue of high-quality topological materials, Nature (London) 566, 480 (2019).

[34] P. Liu, J. R. Williams, and J. J. Cha, Topological nanomaterials, Nat. Rev. Mater. 4, 479 (2019).

[35] J. Tian, I. Miotkowski, S. Hong, and Y. P. Chen, Electrical injection and detection of spin-polarized currents in topological insulator $\mathrm{Bi}_{2} \mathrm{Te}_{2} \mathrm{Se}$, Sci. Rep. 5, 14293 (2015).

[36] J. Tian, S. Hong, I. Miotkowski, S. Datta, and Y. P. Chen, Observation of current-induced, long-lived persistent spin polarization in a topological insulator: A rechargeable spin battery, Sci. Adv. 3, e160253 (2017).

[37] S. Parkin, X. Jiang, C. Kaiser, A. Panchula, K. Roche, and M. Samant, Magnetically engineered spintronic sensors and memory, Proc. IEEE 91, 661 (2003).

[38] G. Gatti, D. Gosálbez-Martínez, S. S. Tsirkin, M. Fanciulli, M. Puppin, S. Polishchuk, S. Moser, L. Testa, E. Martino, S. Roth, P. Bugnon, L. Moreschini, A. Bostwick, C. Jozwiak, E. Rotenberg, G. Di Santo, L. Petaccia, I. Vobornik, J. Fujii, J. Wong et al., Radial Spin Texture of the Weyl Fermions in Chiral Tellurium, Phys. Rev. Lett. 125, 216402 (2020).

[39] Y. Liu, X. Chen, and Y. Xu, Topological phononics: From fundamental models to real materials, Adv. Funct. Mater. 30, 1904784 (2020).

[40] X. S. Wang and X. R. Wang, Topological magnonics, J. Appl. Phys. 129, 151101 (2021).

[41] T. Ozawa, H. M. Price, A. Amo, N. Goldman, M. Hafezi, L. Lu, M. C. Rechtsman, D. Schuster, J. Simon, O. Zilberberg, and I. Carusotto, Topological photonics, Rev. Mod. Phys. 91, 015006 (2019).

[42] C. H. Lee, S. Imhof, C. Berger, F. Bayer, J. Brehm, L. W. Molenkamp, T. Kiessling, and R. Thomale, Topolectrical circuits, Commun. Phys. 1, 39 (2018).

[43] R. Yu, Y. X. Zhao, and A. P. Schnyder, 4D spinless topological insulator in a periodic electric circuit, Natl. Sci. Rev. 7, 1288 (2020).

[44] Z. Wang, D. Gresch, A. A. Soluyanov, W. Xie, S. Kushwaha, X. Dai, M. Troyer, R. J. Cava, and B. A. Bernevig, $\mathrm{MoTe}_{2}$ : A Type-II Weyl Topological Metal, Phys. Rev. Lett. 117, 056805 (2016).

[45] K. Koepernik, D. Kasinathan, D. V. Efremov, S. Khim, S. Borisenko, B. Büchner, and J. van den Brink, TaIrTe ${ }_{4}$ : A ternary type-II Weyl semimetal, Phys. Rev. B 93, 201101(R) (2016).

[46] I. Belopolski, P. Yu, D. S. Sanchez, Y. Ishida, T.-R. Chang, S. S. Zhang, S.-Y. Xu, H. Zheng, G. Chang, G. Bian et al., Signatures of a time-reversal symmetric Weyl semimetal with only four Weyl points, Nat. Commun. 8, 1 (2017).
[47] T. Hahn, The 230 space groups, in International Tables for Crystallography Volume A: Space-group symmetry, edited by T. Hahn (Springer Netherlands, Dordrecht, 2002), pp. 112717.

[48] W. Setyawan and S. Curtarolo, High-throughput electronic band structure calculations: Challenges and tools, Comput. Mater. Sci. 49, 299 (2010).

[49] See Supplemental Material at http://link.aps.org/supplemental/ 10.1103/PhysRevMaterials.5.124202 for screening results of selected SGs.

[50] G. Kresse and J. Furthmüller, Efficient iterative schemes for ab initio total-energy calculations using a plane-wave basis set, Phys. Rev. B 54, 11169 (1996).

[51] G. Kresse and J. Furthmüller, Efficiency of ab-initio total energy calculations for metals and semiconductors using a plane-wave basis set, Comput. Mater. Sci. 6, 15 (1996).

[52] P. E. Blöchl, Projector augmented-wave method, Phys. Rev. B 50, 17953 (1994).

[53] G. Kresse and D. Joubert, From ultrasoft pseudopotentials to the projector augmented-wave method, Phys. Rev. B 59, 1758 (1999).

[54] J. P. Perdew, K. Burke, and M. Ernzerhof, Generalized Gradient Approximation Made Simple, Phys. Rev. Lett. 77, 3865 (1996).

[55] A. A. Mostofi, J. R. Yates, G. Pizzi, Y.-S. Lee, I. Souza, D. Vanderbilt, and N. Marzari, An updated version of wannier90: A tool for obtaining maximally-localised Wannier functions, Comput. Phys. Commun. 185, 2309 (2014).

[56] Q. Wu, S. Zhang, H.-F. Song, M. Troyer, and A. A. Soluyanov, WannierTools : An open-source software package for novel topological materials, Comput. Phys. Commun. 224, 405 (2018).

[57] G. Chang, B. J. Wieder, F. Schindler, D. S. Sanchez, I. Belopolski, S.-M. Huang, B. Singh, D. Wu, T.-R. Chang, T. Neupert et al., Topological quantum properties of chiral crystals, Nat. Mater. 17, 978 (2018).

[58] A. König and N. D. Mermin, Electronic level degeneracy in nonsymmorphic periodic or aperiodic crystals, Phys. Rev. B 56, 13607 (1997).

[59] A. König and N. D. Mermin, Screw rotations and glide mirrors: Crystallography in Fourier space, Proc. Natl. Acad. Sci. 96, 3502 (1999).

[60] L. Michel and J. Zak, Connectivity of energy bands in crystals, Phys. Rev. B 59, 5998 (1999).

[61] Z. Wang, A. Alexandradinata, R. J. Cava, and B. A. Bernevig, Hourglass fermions, Nature (London) 532, 189 (2016).

[62] R. M. Geilhufe, S. S. Borysov, A. Bouhon, and A. V. Balatsky, Data mining for three-dimensional organic Dirac materials: focus on space group 19, Sci. Rep. 7, 1 (2017).

[63] T. Matković and K. Schubert, Kristallstruktur von $\mathrm{Pd}_{7} \mathrm{Se}_{4}$, J. Less Common Metals 59, P57 (1978).

[64] S. Kukunuri, S. N. Karthick, and S. Sampath, Robust, metallic Pd17Se15 and Pd7Se4 phases from a single source precursor and their use as counter electrodes in dye sensitized solar cells, J. Mater. Chem. A 3, 17144 (2015).

[65] T. Olsen, E. Røst, F. Grønvold, A. F. Andresen, E. Hoyer, V. P. Spiridonov, and T. G. Strand, Phase relationships of palladium selenides, Acta Chem. Scand. 33a, 251 (1979).

[66] M. Xiao and S. Fan, Topologically charged nodal surface, arXiv:1709.02363 (2017); M. Xiao, L. Ye, C. Qiu, H. He, 
Z. Liu, and S. Fan, Experimental demonstration of acoustic semimetal with topologically charged nodal surface, Sci. Adv. 6, eaav2360 (2020).

[67] M. A. Wilde, M. Dodenhöft, A. Niedermayr, A. Bauer, M. M. Hirschmann, K. Alpin, A. P. Schnyder, and C. Pfleiderer, Symmetry-enforced topological nodal planes at the Fermi surface of a chiral magnet, Nature (London) 594, 374 (2021).

[68] H. Nielsen and M. Ninomiya, A no-go theorem for regularizing chiral fermions, Phys. Lett. B 105, 219 (1981).

[69] Z.-M. Yu, W. Wu, Y. X. Zhao, and S. A. Yang, Circumventing the no-go theorem: A single Weyl point without surface Fermi arcs, Phys. Rev. B 100, 041118(R) (2019).

[70] F. Flicker, F. de Juan, B. Bradlyn, T. Morimoto, M. G. Vergniory, and A. G. Grushin, Chiral optical response of multifold fermions, Phys. Rev. B 98, 155145 (2018).

[71] C. Herring, Accidental degeneracy in the energy bands of crystals, Phys. Rev. 52, 365 (1937).

[72] G. A. Wiegers, The crystal structure of the low-temperature form of silver selenide, Am. Mineral. 56, 1882 (1971).

[73] L. Wu, F. Tang, and X. Wan, Exhaustive list of topological hourglass band crossings in 230 space groups, Phys. Rev. B 102, 035106 (2020).

[74] W. Wu, Y. Jiao, S. Li, X.-L. Sheng, Z.-M. Yu, and S. A. Yang, Hourglass Weyl loops in two dimensions: Theory and material realization in monolayer GaTeI family, Phys. Rev. Materials 3, 054203 (2019).

[75] W. Klünter, B. Schmidt, and W. Jung, LiIrB, CuIrB und PdIrB, ternäre Iridiumboride mit neuen, vom $\mathrm{CaRh}_{2} \mathrm{~b}_{2}$-Typ abgeleiteten Strukturen, J. Alloys Compd. 205, 93 (1994).

[76] M. A. McGuire, T. K. Reynolds, and F. J. DiSalvo, Crystal structure, electronic structure, and thermoelectric properties of AuTlSb: A new pyrite superstructure, J. Alloys Compd. 425, 81 (2006)

[77] S. M. Young, S. Zaheer, J. C. Y. Teo, C. L. Kane, E. J. Mele, and A. M. Rappe, Dirac Semimetal in Three Dimensions, Phys. Rev. Lett. 108, 140405 (2012).

[78] B.-J. Yang and N. Nagaosa, Classification of stable threedimensional Dirac semimetals with nontrivial topology, Nat. Commun. 5, 4898 (2014).

[79] S.-S. Wang, Y. Liu, Z.-M. Yu, X.-L. Sheng, and S. A. Yang, Hourglass Dirac chain metal in rhenium dioxide, Nat. Commun. 8, 1 (2017).

[80] F. Zhou, G. Ding, Z. Cheng, G. Surucu, H. Chen, and X. Wang, Pnma metal hydride system LiBH: A superior topological semimetal with the coexistence of twofold and quadruple degenerate topological nodal lines, J. Phys.: Condens. Matter 32, 365502 (2020).

[81] D. Shao, H. Wang, T. Chen, P. Lu, Q. Gu, L. Sheng, D. Xing, and J. Sun, Composite topological nodal lines penetrating the Brillouin zone in orthorhombic $\mathrm{AgF}_{2}$, npj Comput. Mater. 5, 1 (2019).

[82] Y. Chen, H.-S. Kim, and H.-Y. Kee, Topological crystalline semimetals in nonsymmorphic lattices, Phys. Rev. B 93, 155140 (2016).

[83] X. Wang, G. Ding, Z. Cheng, X.-L. Wang, G. Zhang, and $\mathrm{T}$. Yang, Intersecting nodal rings in orthorhombictype $\mathrm{BaLi}_{2} \mathrm{Sn}$ compound, J. Mater. Chem. C 8, 5461 (2020).
[84] X. Zhang, L. Jin, X. Dai, G. Chen, and G. Liu, Ideal inner nodal chain semimetals in $\mathrm{Li}_{2} \mathrm{XY}(\mathrm{X}=\mathrm{Ca}, \mathrm{Ba} ; \mathrm{Y}=\mathrm{Si}, \mathrm{Ge})$ materials, J. Phys. Chem. Lett. 9, 5358 (2018).

[85] X. Zhou, C.-H. Hsu, H. Aramberri, M. Iraola, C.-Y. Huang, J. L. Mañes, M. G. Vergniory, H. Lin, and N. Kioussis, Novel family of topological semimetals with butterfly-like nodal lines, Phys. Rev. B 104, 125135 (2021).

[86] S. Li, Y. Liu, S.-S. Wang, Z.-M. Yu, S. Guan, X.-L. Sheng, Y. Yao, and S. A. Yang, Nonsymmorphic-symmetry-protected hourglass Dirac loop, nodal line, and Dirac point in bulk and monolayer $X_{3} \operatorname{SiTe}_{6}(X=\mathrm{Ta}, \mathrm{Nb})$, Phys. Rev. B 97, 045131 (2018).

[87] X. Wang, G. Ding, S. A. Khandy, Z. Cheng, G. Zhang, X.L. Wang, and H. Chen, Unique topological nodal line states and associated exceptional thermoelectric power factor platform in $\mathrm{Nb}_{3} \mathrm{GeTe}_{6}$ monolayer and bulk, Nanoscale 12, 16910 (2020).

[88] Y. Gao, Y. Xie, Y. Chen, J. Gu, and Z. Chen, Spindle nodal chain in three-dimensional $\alpha^{\prime}$ boron, Phys. Chem. Chem. Phys. 20, 23500 (2018).

[89] J. Cano, B. Bradlyn, Z. Wang, L. Elcoro, M. G. Vergniory, C. Felser, M. I. Aroyo, and B. A. Bernevig, Building blocks of topological quantum chemistry: Elementary band representations, Phys. Rev. B 97, 035139 (2018).

[90] G. F. Lange, A. Bouhon, and R.-J. Slager, Subdimensional topologies, indicators, and higher order boundary effects, Phys. Rev. B 103, 195145 (2021).

[91] R. S. K. Mong, A. M. Essin, and J. E. Moore, Antiferromagnetic topological insulators, Phys. Rev. B 81, 245209 (2010).

[92] L. Fu and C. L. Kane, Topological insulators with inversion symmetry, Phys. Rev. B 76, 045302 (2007).

[93] F. Merlo and M. Fornasini, Crystal structure of some phases and alloying behaviour in alkaline earths, europium and ytterbium pnictides, Mater. Res. Bull. 29, 149 (1994).

[94] N. D. Smith, T. Lichtenstein, J. Gesualdi, K. Kumar, and H. Kim, Thermodynamic properties of strontium-bismuth alloys determined by electromotive force measurements, Electrochim. Acta 225, 584 (2017).

[95] K. Schubert, S. Bhan, W. Burkhardt, R. Gohle, H. G. Meissner, M. Pötzschke, and E. Stolz, Einige strukturelle Ergebnisse an metallischen Phasen (5), Sci. Nat. 47, 303 (1960).

[96] L. N. Finnie, Structures and compositions of the silicides of ruthenium, osmium, rhodium and iridium, J. Alloys Compd. 4, 24 (1962).

[97] F. Schindler, A. M. Cook, M. G. Vergniory, Z. Wang, S. S. P. Parkin, B. A. Bernevig, and T. Neupert, Higher-order topological insulators, Sci. Adv. 4, eaat0346 (2018).

[98] W. A. Benalcazar, T. Li, and T. L. Hughes, Quantization of fractional corner charge in $C_{n}$-symmetric higher-order topological crystalline insulators, Phys. Rev. B 99, 245151 (2019).

[99] L. Trifunovic and P. W. Brouwer, Higher-Order BulkBoundary Correspondence for Topological Crystalline Phases, Phys. Rev. X 9, 011012 (2019).

[100] B. J. Wieder, Z. Wang, J. Cano, X. Dai, L. M. Schoop, B. Bradlyn, and B. A. Bernevig, Strong and fragile topological Dirac semimetals with higher-order Fermi arcs, Nat. Commun. 11, 1 (2020). 
[101] H.-X. Wang, Z.-K. Lin, B. Jiang, G.-Y. Guo, and J.-H. Jiang, Higher-Order Weyl Semimetals, Phys. Rev. Lett. 125, 146401 (2020).

[102] K. Wang, J.-X. Dai, L. B. Shao, S. A. Yang, and Y. X. Zhao, Boundary Criticality of $\mathcal{P} \mathcal{T}$-Invariant Topology and Second-
Order Nodal-Line Semimetals, Phys. Rev. Lett. 125, 126403 (2020).

[103] P. M. Lenggenhager, X. Liu, T. Neupert, and T. Bzdušek, Universal higher-order bulk-boundary correspondence of triple nodal points, arXiv:2104.11254 [cond-mat.mes-hall]. 